\title{
Bounds and algorithms for the $K$-Bessel function of imaginary order
}

\author{
Andrew R. Booker, Andreas Strömbergsson and Holger Then
}

\begin{abstract}
Using the paths of steepest descent, we prove precise bounds with numerical implied constants for the modified Bessel function $K_{i r}(x)$ of imaginary order and its first two derivatives with respect to the order. We also prove precise asymptotic bounds on more general (mixed) derivatives without working out numerical implied constants. Moreover, we present an absolutely and rapidly convergent series for the computation of $K_{i r}(x)$ and its derivatives, as well as a formula based on Fourier interpolation for computing with many values of $r$. Finally, we have implemented a subset of these features in a software library for fast and rigorous computation of $K_{i r}(x)$.
\end{abstract}

\section{Introduction}

'If we can qualify a special function as being important when it appears in mathematical and physical applications, then the modified Bessel function of the third kind of imaginary orders is a quite important one' [16]. In mathematics, this function plays an important role in analytic number theory $[\mathbf{5}, \mathbf{1 8}, \mathbf{2 6}, \mathbf{3 5}]$, and in the spectral theory of automorphic forms $[\mathbf{2 2}]$. It appears in the study of harmonic analysis on arithmetic manifolds [21], and in ergodic theory [43]. In physics, we encounter it in arithmetic quantum chaos $[4,39]$, and in cosmology, $K_{i r}(x)$ enters when studying metric perturbations in hyperbolic universes with a horned topology $[2,33]$.

In view of upcoming applications in analytic number theory [6], we need precise bounds with numerical implied constants on $K_{i r}(x)$ and algorithms for its rigorous computation at an accuracy of several hundred decimal places for a vast range of arguments and imaginary orders.

Plenty of literature exists for $K_{i r}(x)[\mathbf{1}, \mathbf{1 4}, \mathbf{2 8}, \mathbf{4 2}]$, some of which presents uniform asymptotic expansions $[\mathbf{3}, \mathbf{1 2}, \mathbf{1 6}]$. In particular, $[\mathbf{3}]$ gives precise bounds on the error terms and one could in principle follow [29] to get quite precise numerical bounds on the error in the asymptotic expansions of $K_{i r}(x)$ and its derivative with respect to $x$. Besides, a whole range of methods have been employed to bring the numerical integration forward $[\mathbf{1 5}, \mathbf{2 3}$ ], for instance, deforming the contour of integration [25], rearranging the oscillatory integrand [24], using Fourier transform methods [9], using the method of steepest descent [19], [20, pp. 117(bottom)-123]. Moreover, a generalized Simpson rule for numerical quadrature of oscillatory integrals was developed [13], a variety of series and continued fraction expansions have been utilized $[\mathbf{1 0}, \mathbf{1 1}, \mathbf{4 1}]$, and hyperasymptotic expansions established [32].

However, we could not locate a reference that readily satisfies our demanding requests concerning precise bounds with explicit numerical implied constants on $K_{i r}(x)$ and its derivatives. In particular in the forthcoming work [6] we also need precise bounds on the

Received 18 June 2012; revised 3 October 2012.

2010 Mathematics Subject Classification 26D07, 33C10, 33F05, 34D05, 41A58 (primary), 41A80, 65D05, 40H05, 26B99 (secondary).

A. B. and H. T. are supported by EPSRC Fellowship EP/H005188/1. A. S. is a Royal Swedish Academy of Sciences Research Fellow supported by a grant from the Knut and Alice Wallenberg Foundation. 
derivatives of $K_{i r}(x)$ with respect to the order, and on mixed derivatives; such bounds are underrepresented in the literature and we aim to close this gap.

While better and better algorithms for computing higher transcendental functions become available, they still seem to be off from our goals of being highly accurate, rigorous, and fast. Difficulties arise, especially when the imaginary order of $K_{i r}(x)$ becomes large. We seek to advance the subject by deriving absolute and rapidly convergent series for $K_{i r}(x)$, and to boost the speed of rigorous high accuracy computations by Fourier interpolation.

The modified Bessel function of the third kind is defined by

$$
K_{i r}(x)=\frac{1}{2} \int_{-\infty}^{\infty} e^{\phi(t)} d t \quad \text { where } \phi(t):=-x \cosh t+i r t
$$

see [42, p. 181]. It satisfies the modified Bessel differential equation

$$
x^{2} y^{\prime \prime}+x y^{\prime}+\left(r^{2}-x^{2}\right) y=0
$$

and decays exponentially for large arguments

$$
K_{i r}(x) \sim \sqrt{\frac{\pi}{2 x}} e^{-x} \text { for } x \rightarrow \infty .
$$

A second linearly independent solution of the differential equation is the modified Bessel function of the first kind

$$
I_{i r}(x)=\left(\frac{x}{2}\right)^{i r} \sum_{j=0}^{\infty} \frac{(x / 2)^{2 j}}{j ! \Gamma(1+j+i r)},
$$

which grows exponentially for large arguments

$$
I_{i r}(x) \sim \sqrt{\frac{1}{2 \pi x}} e^{x} \quad \text { for } x \rightarrow \infty .
$$

We assume that $r>0, x>0$. While $I_{i r}(x)$ is complex, $K_{i r}(x)$ is real and an even function with respect to $r$. In fact, it is the imaginary part of $I_{i r}(x)$, up to a factor,

$$
\operatorname{Im} I_{i r}(x)=-\frac{\sinh \pi r}{\pi} K_{i r}(x) .
$$

Guided by an unpublished manuscript of Hejhal [19] and by the literature [37], we use the paths of steepest descent to convert (1) into non-oscillatory integrals. For reasons of convenience, however, we deviate from some piece of the path of steepest descent and replace it by a simpler one on which the absolute value of the integrand is sufficiently small [19]. Exponential bounds on the integrands as well as the resulting bounds on $K_{i r}(x)$ and its derivatives are stated in $\S 2$ and are proven in the appendix. Section 3 focuses on the computational aspects of $K_{i r}(x)$. Applying the Poisson summation formula to the imaginary part of the power series (3) results in an absolutely and rapidly convergent series for $K_{i r}(x)$, which, by bounding the exponentially small truncation errors, serves as an algorithm for the rigorous high-accuracy computation of $K_{i r}(x)$ and its derivatives. We also describe a second algorithm based on Fourier interpolation for computing $K_{i r}(x)$ for fixed $x$ and many values of $r$. Finally, a subset of these findings has been implemented and can be downloaded as a software library from $[8]$. 


\section{Bounds}

\subsection{Paths of steepest descent}

The saddle point contours of (1) can be found in Temme [37] and we recapitulate them here. Saddle points follow from solving the equation $\phi^{\prime}(t)=0$ which yields

$$
\begin{array}{ll}
t_{n}=i\left((-1)^{n} \arcsin \left(\frac{r}{x}\right)+n \pi\right), & n \in \mathbb{Z}, \text { if } r \leqslant x, \\
t_{n}^{ \pm}= \pm \operatorname{arcosh}\left(\frac{r}{x}\right)+i \pi\left(2 n+\frac{1}{2}\right), & n \in \mathbb{Z}, \text { if } r \geqslant x .
\end{array}
$$

2.1.1. The monotonic case: $x \geqslant r>0$. In this case we set

$$
\alpha:=\arcsin \left(\frac{r}{x}\right) \in\left(0, \frac{\pi}{2}\right],
$$

and it suffices to consider the saddle point $t_{0}=i \alpha$. The path of steepest descent is defined by the equation $\operatorname{Im} \phi(t)=\operatorname{Im} \phi\left(t_{0}\right)$ which gives

$$
t=: u+i v \quad \text { where } \quad v(u)=\arcsin \left(\sin \alpha \frac{u}{\sinh u}\right), \quad-\infty<u<\infty .
$$

Integrating with respect to this path yields the representation

$$
K_{i r}(x)=\frac{1}{2} \int_{-\infty}^{\infty} e^{\phi(u+i v(u))} \frac{d t}{d u} d u=\int_{0}^{\infty} e^{\eta(u)} d u
$$

where $\eta(u):=\phi(u+i v(u))=-x \cosh u \cos v(u)-r v(u)$ (see [37, equation (2.7)]).

2.1.2. The oscillatory case: $0<x<r$. In this case we set

$$
\mu:=\operatorname{arcosh}\left(\frac{r}{x}\right)>0 \text {. }
$$

The saddle point contour through the saddle $t_{n}^{ \pm}$is defined by the equation $\operatorname{Im} \phi(t)=\operatorname{Im} \phi\left(t_{n}^{ \pm}\right)$ which results in

$$
t=: u+i v \quad \text { with } \sin v=\frac{T u \mp S}{\sinh u},
$$

where $T:=r / x=\cosh \mu>1, S:=\mu \cosh \mu-\sinh \mu>0$. Note that the dependence on $n$ is implicit upon solving for $v(u)$.

The path of steepest descent is a countable union of pieces of saddle point contours (5) and runs from $-\infty$ through the saddle points $\left\{t_{n}^{-}\right\}_{n \geqslant 0}$ up to $i \infty$ and from there symmetrically down through the saddle points $\left\{t_{n}^{+}\right\}_{n \geqslant 0}$ to $+\infty$, see [37]. Since $r>0$, the integrand $e^{\phi(t)}$ is exponentially small in $v$ and vanishes at $i \infty$. Using the path of steepest descent results in the integral representations [37, equations (3.3), (3.5)] which could be used to bound $K_{i r}(x)$.

However, when proving our bounds we have found it more convenient to not follow the path of steepest descent all the way up to $i \infty$, but to use the pieces with imaginary part less than some positive constant only, and to replace the omitted part by a straight line [19]. The price to pay for this simplification is that we will not bound $K_{i r}(x)$ for $x<1$. (Fortunately, other representations of $K_{i r}(x)$, such as the series (25), are easy to bound for $x<1$; see the proof of Proposition 5 on page 106 for an example of this in practice.)

We set $u_{\pi}:=S / T>0$. Then the path of steepest descent for $u \geqslant u_{\pi}$ reads $t=u+i v$ with

$$
v(u)= \begin{cases}\pi-\arcsin \left(\frac{T u-S}{\sinh u}\right) & \text { if } u \in\left[u_{\pi}, \mu\right], \\ \arcsin \left(\frac{T u-S}{\sinh u}\right) & \text { if } u \in[\mu, \infty) .\end{cases}
$$


One checks by differentiation that $v(u)$ is strictly decreasing for all $u \in\left[u_{\pi}, \infty\right)$. We remark that $v(u)$ is smooth for all $u>u_{\pi}$; the fact that it is smooth at $u=\mu$ follows from the construction and basic principles of complex analysis. Note also the special values $v\left(u_{\pi}\right)=\pi$, $v(\mu)=\pi / 2$, and $v^{\prime}(\mu)=-1$; the last identity follows for example from the fact that $\phi^{\prime \prime}\left(t_{0}^{+}\right)=$ $-i \sqrt{r^{2}-x^{2}}$, a negative imaginary number.

If we now fix some $u_{c} \in\left[u_{\pi}, \mu\right]$, we can define

$$
t_{c}(u):= \begin{cases}u+i v(|u|) & \text { if }|u| \geqslant u_{c}, \\ u+i v\left(u_{c}\right) & \text { if }|u|<u_{c},\end{cases}
$$

which is a continuous path from $-\infty$ to $+\infty$. If $u \neq \pm u_{c}$ the path is smooth, and for $|u| \geqslant u_{c}$ it coincides with the path of steepest descent. Replacing in (1) the contour of integration by the path $t_{c}(u)$ results in the representation

$$
K_{i r}(x)=\frac{1}{2} \int_{-\infty}^{\infty} e^{\phi\left(t_{c}(u)\right)} \frac{d t_{c}}{d u} d u .
$$

The justification of this step via Cauchy's integral theorem is easy, since $\lim _{u \rightarrow \infty} v(u)=0$ and $\operatorname{Re} \phi(u+i v)=-x \cosh u \cos v-r v$ is rapidly decaying as $u \rightarrow \pm \infty$, uniformly with respect to $v$ in any compact subset of $[0, \pi / 2)$ (and $x, r$ fixed).

Utilizing the symmetries $t_{c}(-u)=-\overline{t_{c}(u)}$ and $\phi(-\bar{t})=\overline{\phi(t)}$, we arrive at the integral representation

$$
K_{i r}(x)=\operatorname{Re}\left\{\int_{0}^{u_{c}} e^{\phi\left(u+i v\left(u_{c}\right)\right)} d u+\int_{u_{c}}^{\infty} e^{\phi(u+i v(u))}\left(1+i v^{\prime}(u)\right) d u\right\},
$$

which we are going to bound.

\subsection{Bounds}

2.2.1. The monotonic case: $x \geqslant r>0$. The integrand of (4) reads $e^{\eta(u)}$ with

$$
\eta(u)=-x \cosh u \cos v(u)-r v(u) .
$$

By construction, we know that $\eta(u)$ has a maximum at $u=0$ and we easily compute

$$
\eta(0)=-x \cos \alpha-r \alpha, \quad \eta^{\prime}(0)=0, \quad \eta^{\prime \prime}(0)=-x \cos \alpha=-\sqrt{x^{2}-r^{2}} .
$$

It turns out that $\eta(u)$ lies below the parabola described by $\eta(0), \eta^{\prime}(0), \eta^{\prime \prime}(0)$.

Lemma 1. Assume $x \geqslant r>0$. Then for all $u \in \mathbb{R}$ we have

$$
\eta(u) \leqslant-x \cos \alpha-r \alpha-\frac{1}{2} \sqrt{x^{2}-r^{2}} u^{2} .
$$

The proof is given in the appendix on page 89 .

In the case of $x / r$ near 1 , we also need another bound, to show that once $u$ gets larger than 0 by a not too small amount, $\eta(u)$ decays quite a bit more rapidly than what is given by Lemma 1. To appreciate the following lemma, note that for any fixed $x=r>0$, we have,

$$
\begin{aligned}
\eta(u) & =-r\left(\cosh u \sqrt{1-\frac{u^{2}}{\sinh ^{2} u}}+\arcsin \left(\frac{u}{\sinh u}\right)\right) \\
& =-\frac{\pi}{2} r-\frac{4 \sqrt{3}}{27} r u^{3}+O\left(u^{7}\right) \quad \text { as } u \rightarrow 0^{+} .
\end{aligned}
$$

Lemma 2. Assume $x \geqslant r>0$. Then for all $u \geqslant 0$ we have

$$
\eta(u) \leqslant-x \cos \alpha-r \alpha-\frac{4 \sqrt{3}}{27} r u^{3} .
$$

The proof is given in the appendix on page 90 .

For bounding the partial derivatives of $K_{i r}(x)$ we will also need bounds on $v^{\prime}(u)$. 
Lemma 3 (cf. [19]). Assume $x \geqslant r>0$. Then for all $u>0$ we have

$$
0>v^{\prime}(u)>-3^{-\frac{1}{2}}
$$

and

$$
0>u v^{\prime}(u)>-\sqrt{3}
$$

The proof is given in the appendix on page 90 .

Based on (4) and Lemmata 1-3, we can bound $K_{i r}(x)$ and its derivatives for $x \geqslant r>0$.

Proposition 1. For all $x \geqslant r>0$ we have:

$$
\begin{gathered}
0<K_{i r}(x) \leqslant e^{-(\pi / 2) r} e^{-\sqrt{x^{2}-r^{2}}+r \arccos (r / x)} \min \left(\frac{\sqrt{\pi / 2}}{\sqrt[4]{x^{2}-r^{2}}}, \frac{\Gamma\left(\frac{1}{3}\right)}{2^{\frac{2}{3}} 3^{\frac{1}{6}}} r^{-\frac{1}{3}}\right), \\
\left|\frac{\partial}{\partial r} K_{i r}(x)\right|<e^{-(\pi / 2) r} e^{-\sqrt{x^{2}-r^{2}}+r \arccos (r / x)} \min \left(\frac{\sqrt{3 \pi / 2}}{\sqrt[4]{x^{2}-r^{2}}}, \frac{3^{\frac{1}{3}} \Gamma\left(\frac{1}{3}\right)}{2^{\frac{2}{3}}} r^{-\frac{1}{3}}\right),
\end{gathered}
$$

and

$$
\begin{aligned}
\left|\frac{\partial^{2}}{\partial r^{2}} K_{i r}(x)\right|< & e^{-(\pi / 2) r} e^{-\sqrt{x^{2}-r^{2}}+r \arccos (r / x)} \\
& \times \min \left(\frac{\frac{1}{2} \pi^{\frac{3}{2}}\left(\sqrt{3}-\frac{\pi}{4}\right)}{\left(x^{2}-r^{2}\right)^{1 / 4}}+\frac{\sqrt{\pi / 2}}{\left(x^{2}-r^{2}\right)^{3 / 4}}, \frac{\pi\left(\sqrt{3}-\frac{\pi}{4}\right) \Gamma\left(\frac{1}{3}\right)}{2^{\frac{2}{3}} 3^{\frac{1}{6}}} r^{-\frac{1}{3}}+\frac{3^{\frac{3}{2}}}{4} r^{-1}\right) .
\end{aligned}
$$

Furthermore, for any fixed integers $j_{1}, j_{2} \geqslant 0$ and any $\varepsilon>0$, the following holds for all $r>0$, $x \geqslant \max (\varepsilon, r)$ :

$$
\left|\frac{\partial^{j_{1}+j_{2}}}{\partial r^{j_{1}} \partial x^{j_{2}}} K_{i r}(x)\right| \ll_{j_{1}, j_{2}, \varepsilon} e^{-(\pi / 2) r} e^{-\sqrt{x^{2}-r^{2}}+r \arccos (r / x)} \frac{\max \left(\sqrt[4]{x^{2}-r^{2}}, r^{\frac{1}{3}}\right)^{2 j_{2}-1}}{x^{j_{2}}} .
$$

The proof is given in the appendix on page 90 . We remark that $a \ll_{\mu_{1}, \mu_{2}, \ldots} b$ means that there exists a positive function $C\left(\mu_{1}, \mu_{2}, \ldots\right)$, independent of all other variables, such that $|a| \leqslant C\left(\mu_{1}, \mu_{2}, \ldots\right) b$ holds true.

2.2.2. The oscillatory case: $0<x<r$. For $u \geqslant u_{\pi}$, we study $\eta(u):=\operatorname{Re} \phi(u+i v(u))$ along the curve (6), (7). As before,

$$
\eta(u)=-x \cosh u \cos v(u)-r v(u) .
$$

This function has a maximum at $u=\mu$ and we compute

$$
\eta(\mu)=-\frac{\pi}{2} r, \quad \eta^{\prime}(\mu)=0, \quad \eta^{\prime \prime}(\mu)=-2 x \sinh \mu=-2 \sqrt{r^{2}-x^{2}} .
$$

Lemma 4. Assume $0<x<r$. Then for all $u \geqslant u_{\pi}$ we have

$$
\eta(u) \leqslant-\frac{\pi}{2} r-\sqrt{r^{2}-x^{2}}(u-\mu)^{2},
$$

and for all $u \geqslant \mu$ :

$$
\eta(u) \leqslant-\frac{\pi}{2} r-\frac{4 \sqrt{3}}{27} r(u-\mu)^{3}
$$

The proof is given in the appendix on page 92 . Note that the constant $4 \sqrt{3} / 27$ in (20) is also optimal, as is seen by considering the limiting case $\mu=0$ (cf. (10)).

For bounding $K_{i r}(x)$ we need bounds on $v^{\prime}(u)$ as well. As we have already pointed out, $v(u)$ is strictly decreasing, and in particular $v^{\prime}(u) \leqslant 0$ for all $u \geqslant u_{\pi}$. 
Lemma 5. Assume $0<x<r$. Then:

(a) $v^{\prime}(u)$ is strictly increasing for all $u \geqslant u_{\pi}$;

(b) if $0<\mu \leqslant 1.8$ then $-2.9<v^{\prime}(u) \leqslant-1$ for all $u \in\left[\frac{1}{2} \mu, \mu\right]$;

(c) if $\mu \geqslant 1.8$ then $-3.3<v^{\prime}(u) \leqslant-1$ for all $u \in\left[u_{\pi}, \mu\right]$.

The proof is given in the appendix page 97 .

Based on (8) and Lemmata 4 and 5 , we can bound $K_{i r}(x)$ and its derivatives for $1 \leqslant x<r$.

Proposition 2. For all $1 \leqslant x<r$ we have

$$
\begin{gathered}
\left|K_{i r}(x)\right|<e^{-(\pi / 2) r} \begin{cases}\frac{5}{\sqrt[4]{r^{2}-x^{2}}} & \text { if } x \leqslant r-\frac{1}{2} r^{\frac{1}{3}}, \\
4 r^{-\frac{1}{3}} & \text { if } x \geqslant r-\frac{1}{2} r^{\frac{1}{3}},\end{cases} \\
\left|\frac{\partial}{\partial r} K_{i r}(x)\right|<e^{-(\pi / 2) r} \begin{cases}\frac{17+5 \log (r / x)}{\sqrt[4]{r^{2}-x^{2}}} & \text { if } x \leqslant r-\frac{1}{2} r^{\frac{1}{3}}, \\
12 r^{-\frac{1}{3}} & \text { if } x \geqslant r-\frac{1}{2} r^{\frac{1}{3}},\end{cases}
\end{gathered}
$$

and

$$
\left|\frac{\partial^{2}}{\partial r^{2}} K_{i r}(x)\right|<e^{-(\pi / 2) r} \begin{cases}\frac{44+8 \log (r / x)^{2}}{\sqrt[4]{r^{2}-x^{2}}} & \text { if } x \leqslant r-\frac{1}{2} r^{\frac{1}{3}}, \\ 22 r^{-\frac{1}{3}} & \text { if } x \geqslant r-\frac{1}{2} r^{\frac{1}{3}} .\end{cases}
$$

Furthermore, for any fixed integers $j_{1}, j_{2} \geqslant 0$ and any $\varepsilon>0$, the following holds for all $r>x \geqslant \varepsilon$ :

$$
\left|\frac{\partial^{j_{1}+j_{2}}}{\partial r^{j_{1}} \partial x^{j_{2}}} K_{i r}(x)\right| \ll_{j_{1}, j_{2}, \varepsilon} e^{-(\pi / 2) r} \frac{\max \left(\sqrt[4]{r^{2}-x^{2}}, r^{\frac{1}{3}}\right)^{2 j_{2}-1}}{x^{j_{2}}}\left(\log \frac{2 r}{x}\right)^{j_{1}} .
$$

The proof is given in the appendix on page 99 .

We remark that while the constant ' $\sqrt{\pi / 2}$ ' in the bound on $K_{i r}(x)$ in Proposition 1 is optimal in the limit of large $r$ (cf. [3]), the constant ' 5 ' in the first bound in Proposition 2 is about twice the asymptotically optimal constant $\sqrt{2 \pi}$. Note that it would be possible to use Lemma 4 to prove a sharper, but more complicated, bound of the form $e^{-(\pi / 2) r} \sqrt{2 \pi} / \sqrt[4]{r^{2}-x^{2}}$ plus an explicit correction term (of lower order of magnitude as $r \rightarrow \infty$ and $(r-x) / r^{1 / 3} \rightarrow \infty$ ); the main work necessary to obtain such a bound would be to replace Lemma 5 by an explicit bound on $v^{\prime \prime}(u)$ for $u \leqslant \mu$.

\section{Absolutely convergent series}

\subsection{Small argument}

Taking the imaginary part of the power series (3) results in an absolutely convergent series for $K_{i r}(x)$,

$$
e^{(\pi / 2) r} K_{i r}(x)=\frac{\pi e^{(\pi / 2) r}}{\sinh (\pi r)} \operatorname{Im}\left[\sum_{j=0}^{\infty} \frac{-(x / 2)^{i r+2 j}}{j ! \Gamma(1+i r+j)}\right], \quad \forall x>0 .
$$

Using one term of Stirling's expansion we have $\Gamma(s)=(2 \pi)^{\frac{1}{2}} s^{s-\frac{1}{2}} e^{-s} e^{R(s)}$ where $|R(s)| \leqslant$ 1/(6|s|) [30, p. 294]. Hence

$$
\begin{aligned}
|j ! \Gamma(1+i r+j)| \geqslant & 2 \pi(j+1)^{j+1 / 2}|j+1+i r|^{j+1 / 2} e^{-r \arg (j+1+i r)} \\
& \times e^{-2(j+1)} e^{-1 /(6(j+1))-1 /(6|j+1+i r|)}
\end{aligned}
$$


Using this, we can bound the contribution from all terms with $j \geqslant J$ in (25) as follows, writing $X=(x e / 2)^{2} /((J+1)|J+1+i r|)$ and assuming $X<1$ :

$$
\frac{\pi e^{(\pi / 2) r}}{\sinh (\pi r)}\left|\operatorname{Im}\left[\sum_{j=J}^{\infty} \frac{-(x / 2)^{i r+2 j}}{j ! \Gamma(1+i r+j)}\right]\right|<\frac{11}{(J+1)\left(1-e^{-2 \pi r}\right)} \frac{X^{J}}{1-X} .
$$

Hence, for sufficiently small $x$ the series (25) converges rapidly.

For large $x$, however, $I_{i r}(x)$ increases exponentially, while $K_{i r}(x)$ decreases exponentially. This discrepancy results in catastrophic cancellation of significant digits in the summation of the finite parts of (25), making this series unsuitable once $x$ becomes large. This can be overcome by employing Poisson summation.

\subsection{Large argument}

Let $F: \mathbb{R} \rightarrow \mathbb{C}$ be a Schwartz function, and define $\hat{F}(\xi)=(1 / 2 \pi) \int_{\mathbb{R}} F(t) e^{-i \xi t} d t$. Further let $B>0$ and $t_{0} \in \mathbb{R}$. Then the Poisson sum formula yields

$$
\sum_{k \in \mathbb{Z}} F\left(t_{0}+2 \pi k B\right)=\frac{1}{B} \sum_{n \in \mathbb{Z}} e^{-i n t_{0} / B} \hat{F}\left(-\frac{n}{B}\right) .
$$

Let us apply this with $F(t):=F_{s}(t):=e^{(\pi / 2) r+s t} K_{i r}\left(e^{t}\right)$ for some fixed $s \in \mathbb{C}$ with $\operatorname{Re}(s)>0$. We obtain

$$
\begin{aligned}
\hat{F}_{s}(\xi) & =\frac{e^{(\pi / 2) r}}{2 \pi} \int_{\mathbb{R}} K_{i r}\left(e^{t}\right) e^{(s-i \xi) t} d t=\frac{e^{(\pi / 2) r}}{2 \pi} \int_{0}^{\infty} K_{i r}(x) x^{s-i \xi} \frac{d x}{x} \\
& =\frac{e^{(\pi / 2) r}}{2 \pi} g(s-i \xi+i r) g(s-i \xi-i r),
\end{aligned}
$$

where $g(z):=2^{z / 2-1} \Gamma(z / 2)$. Thus, $(27)$ reads

$$
\sum_{k \in \mathbb{Z}} F_{s}\left(t_{0}+2 \pi k B\right)=\frac{e^{(\pi / 2) r}}{2 \pi B} \sum_{n \in \mathbb{Z}} e^{-i n t_{0} / B} g\left(s+i r+\frac{i n}{B}\right) g\left(s-i r+\frac{i n}{B}\right) .
$$

Next, from (25), we have

$$
F_{s}(t)=\frac{\pi i e^{(\pi / 2) r} 2^{s-1}}{\sinh (\pi r)}\left[\sum_{j=0}^{\infty} \frac{\left(e^{t} / 2\right)^{s+i r+2 j}}{j ! \Gamma(1+i r+j)}-\sum_{j=0}^{\infty} \frac{\left(e^{t} / 2\right)^{s-i r+2 j}}{j ! \Gamma(1-i r+j)}\right] .
$$

Summing this over $t=t_{0}-2 \pi k B$ for $k=1,2, \ldots$, we obtain

$$
\begin{aligned}
\sum_{k=1}^{\infty} F_{s}\left(t_{0}-2 \pi k B\right)=\frac{\pi i e^{(\pi / 2) r} 2^{s-1}}{\sinh (\pi r)}\left[\sum_{j=0}^{\infty} \frac{\left(e^{t_{0}} / 2\right)^{s+i r+2 j}}{j ! \Gamma(1+i r+j)} \frac{1}{e^{2 \pi B(s+i r+2 j)}-1}\right. \\
\left.-\sum_{j=0}^{\infty} \frac{\left(e^{t_{0}} / 2\right)^{s-i r+2 j}}{j ! \Gamma(1-i r+j)} \frac{1}{e^{2 \pi B(s-i r+2 j)}-1}\right]
\end{aligned}
$$

and substituting into (28), this proves the following lemma.

Lemma 6. For $r>0, s \in \mathbb{C}$ with $\operatorname{Re}(s)>0, t_{0} \in \mathbb{R}, B>0$, we have

$$
\begin{aligned}
\sum_{k=0}^{\infty} F_{s}\left(t_{0}+2 \pi k B\right)= & \frac{e^{(\pi / 2) r}}{2 \pi B} \sum_{n \in \mathbb{Z}} e^{-i n t_{0} / B} g\left(s+i r+\frac{i n}{B}\right) g\left(s-i r+\frac{i n}{B}\right) \\
& -\frac{\pi i e^{(\pi / 2) r} 2^{s-1}}{\sinh (\pi r)}\left[\sum_{j=0}^{\infty} \frac{\left(e^{t_{0}} / 2\right)^{s+i r+2 j}}{j ! \Gamma(1+i r+j)} \frac{1}{e^{2 \pi B(s+i r+2 j)}-1}\right. \\
& \left.-\sum_{j=0}^{\infty} \frac{\left(e^{t_{0}} / 2\right)^{s-i r+2 j}}{j ! \Gamma(1-i r+j)} \frac{1}{e^{2 \pi B(s-i r+2 j)}-1}\right] .
\end{aligned}
$$


Although this was derived for $\operatorname{Re}(s)>0$ only, we see that both sides continue to meromorphic functions of $s$ on all of $\mathbb{C}$, and hence the formula must be true everywhere. Particularly nice values are $s=0$ and $s=1$. With the latter, we set $x=e^{t_{0}}$, divide by $x$ and substitute the definition of $g(z)$, which proves the following proposition.

Proposition 3. For $r>0, x>0, B>0$, we have

$$
\begin{aligned}
\sum_{k=0}^{\infty} e^{(\pi / 2) r+2 \pi k B} K_{i r}\left(x e^{2 \pi k B}\right) & \\
= & \frac{e^{(\pi / 2) r}}{4 \pi B x} \sum_{n \in \mathbb{Z}}\left(\frac{x}{2}\right)^{-i n / B} \Gamma\left(\frac{1}{2}+\frac{i}{2}\left(\frac{n}{B}+r\right)\right) \Gamma\left(\frac{1}{2}+\frac{i}{2}\left(\frac{n}{B}-r\right)\right) \\
& +\frac{\pi e^{(\pi / 2) r}}{\sinh (\pi r)} \operatorname{Im}\left[\sum_{j=0}^{\infty} \frac{(x / 2)^{i r+2 j}}{j ! \Gamma(1+i r+j)} \frac{1}{e^{2 \pi B(2 j+1+i r)}-1}\right] .
\end{aligned}
$$

Using the trivial inequality $\left|K_{i r}(x)\right|<\sqrt{\pi / 2 x} e^{-x}$, for $x \geqslant 1$ and $B \geqslant 1 / 2 \pi$ we have $\left|\sum_{k=1}^{\infty} e^{(\pi / 2) r+2 \pi k B} K_{i r}\left(x e^{2 \pi k B}\right)\right| \leqslant \sqrt{\frac{\pi}{2 x}} e^{(\pi / 2) r} \sum_{k=1}^{\infty} e^{\pi k B-x \exp (2 \pi k B)}<\frac{2}{\sqrt{x}} e^{(\pi / 2) r+\pi B-x \exp (2 \pi B)}$.

Hence, by taking $B$ sufficiently large, (29) can be used to compute an approximation of $e^{(\pi / 2) r} K_{i r}(x)$ to any desired level of accuracy.

Suppose now that we sum the terms of the right-hand side for $|n| \leqslant N$. Using the inequality

$$
\left|\Gamma\left(\frac{1}{2}+i t\right)\right|=\sqrt{\frac{\pi}{\cosh (\pi t)}} \leqslant \sqrt{2 \pi} e^{-\pi|t| / 2},
$$

the terms with $|n|>N$ are bounded as follows:

$$
\begin{aligned}
& \frac{e^{(\pi / 2) r}}{4 \pi B x}\left|\sum_{|n|>N}\left(\frac{x}{2}\right)^{-i n / B} \Gamma\left(\frac{1}{2}+\frac{i}{2}\left(\frac{n}{B}+r\right)\right) \Gamma\left(\frac{1}{2}+\frac{i}{2}\left(\frac{n}{B}-r\right)\right)\right| \\
& \leqslant \frac{e^{(\pi / 2) r}}{2 B x} \sum_{|n|>N} e^{-\pi|n| / 2 B}<\frac{2}{\pi x} e^{(\pi / 2) r-\pi N / 2 B} .
\end{aligned}
$$

Utilizing (26), for $B \geqslant 1 / 2 \pi$ we can truncate the sum over $j$ and bound the terms with $j \geqslant J$,

$$
\frac{\pi e^{(\pi / 2) r}}{\sinh (\pi r)}\left|\operatorname{Im}\left[\sum_{j=J}^{\infty} \frac{(x / 2)^{i r+2 j}}{j ! \Gamma(1+i r+j)} \frac{1}{e^{2 \pi B(2 j+1+i r)}-1}\right]\right|<\frac{17}{(J+1)\left(1-e^{-2 \pi r}\right) e^{2 \pi B}} \frac{X^{J}}{1-X}
$$

assuming $X=(x e / 2)^{2} e^{-4 \pi B} /((J+1)|J+1+i r|)<1$. If we take $B \geqslant(1 / 2 \pi) \max (1, \log (x / 2))$, the series (29) converges rapidly for $x \geqslant 1$.

Summing the finite part of (29) can result in significant cancellation of terms. However, the point is that the terms of (29) do not grow exponentially large (as they do in (25)), so even though there is cancellation, we can use the formula to achieve a fixed absolute accuracy without substantially increasing the precision.

We have implemented a numerical software library for computing $K_{i r}(x)$ which can be downloaded from [8]. For $x \leqslant 2$ it uses (25) and for $x>2$ it uses (29). We take rigorous control over the error when summing the terms in the finite part. For this, we increase the number of digits suitably and sum up using interval arithmetic [27].

Formulas (25) and (29) were derived for $r>0$. Because $K_{i r}(x)$ is an even function with respect to $r$, it is straightforward to compute it for $r<0$ as well. Values of $r$ very near 0 are more cumbersome to deal with, since the truncation bounds given above blow up as $r \rightarrow 0$. Fortunately, we can side-step this problem using the algorithm presented in $\S 3.4$. 


\subsection{Derivatives}

Using the inequality $\left|\partial K_{\text {ir }}(x) / \partial x\right|<\sqrt{\pi / 2 x} e^{-x}(1+1 / x)$, for $x \geqslant 1$ and $B \geqslant 1 / 2 \pi$ we have

$$
\begin{aligned}
& \left|\frac{\partial}{\partial x} \sum_{k=1}^{\infty} e^{(\pi / 2) r+2 \pi k B} K_{i r}\left(x e^{2 \pi k B}\right)\right| \\
& \quad \leqslant \sqrt{\frac{\pi}{2 x}} e^{(\pi / 2) r} \sum_{k=1}^{\infty} e^{3 \pi k B-x \exp (2 \pi k B)}\left(1+\frac{1}{x} e^{-2 \pi k B}\right)<\sqrt{\frac{2 \pi}{x}} e^{(\pi / 2) r+3 \pi B-x \exp (2 \pi B)} .
\end{aligned}
$$

This, together with taking the derivative with respect to $x$ on both sides of (29), can be used to compute an approximation of $\partial K_{i r}(x) / \partial x$ to any desired level of accuracy. Here the terms with $|n|>N$ are bounded as follows:

$$
\begin{aligned}
& \frac{e^{(\pi / 2) r}}{8 \pi B}\left|\sum_{|n|>N} \frac{-i n / B-1}{2}\left(\frac{x}{2}\right)^{-i n / B-2} \Gamma\left(\frac{1}{2}+\frac{i}{2}\left(\frac{n}{B}+r\right)\right) \Gamma\left(\frac{1}{2}+\frac{i}{2}\left(\frac{n}{B}-r\right)\right)\right| \\
& <\frac{2}{\pi x^{2}}\left(\frac{N}{B}+2\right) e^{(\pi / 2) r-\pi N / 2 B}
\end{aligned}
$$

and for $B \geqslant 1 / 2 \pi$ we obtain

$$
\frac{\pi e^{(\pi / 2) r}}{\sinh (\pi r)}\left|\operatorname{Im}\left[\sum_{j=J}^{\infty} \frac{\frac{i r+2 j}{2}(x / 2)^{i r+2 j-1}}{j ! \Gamma(1+i r+j)} \frac{1}{e^{2 \pi B(2 j+1+i r)}-1}\right]\right|<\frac{17}{x e^{2 \pi B}} \frac{\left(\frac{r}{J+1}\right)^{1 / 2}+2}{1-e^{-2 \pi r}} \frac{X^{J}}{1-X},
$$

assuming $X=(x e / 2)^{2} e^{-4 \pi B} /((J+1)|J+1+i r|)<1$. If we take $B \geqslant(1 / 2 \pi) \max (1, \log (x / 2))$, the series converges rapidly for $x \geqslant 1$.

For small arguments, we take the derivative of $(25)$ on both sides and get

$$
e^{(\pi / 2) r} \frac{\partial}{\partial x} K_{i r}(x)=\frac{\pi e^{(\pi / 2) r}}{\sinh (\pi r)} \operatorname{Im}\left[\sum_{j=0}^{\infty} \frac{-\frac{i r+2 j}{2}(x / 2)^{i r+2 j-1}}{j ! \Gamma(1+i r+j)}\right], \quad \forall x>0 .
$$

Using the bound

$$
\frac{\pi e^{(\pi / 2) r}}{\sinh (\pi r)}\left|\operatorname{Im}\left[\sum_{j=J}^{\infty} \frac{-\frac{i r+2 j}{2}(x / 2)^{i r+2 j-1}}{j ! \Gamma(1+i r+j)}\right]\right|<\frac{11}{x} \frac{\left(\frac{r}{J+1}\right)^{1 / 2}+2}{1-e^{-2 \pi r}} \frac{X^{J}}{1-X},
$$

with $X=(x e / 2)^{2} /((J+1)|J+1+i r|)<1$, we see that this series converges rapidly for sufficiently small $x$.

Higher derivatives and integrals of $K_{i r}(x)$ with respect to $x$ follow recursively from the differential equation (2) upon inserting the values of $K_{i r}(x)$ and $\partial K_{i r}(x) / \partial x$ as initial conditions, while derivatives and integrals with respect to $r$ are best computed from the formulas given in the next section.

\subsection{Fourier interpolation}

The algorithms described in $\S \S 3.2$ and 3.3 are fast when one is interested in computing $K_{i r}(x)$ for a fixed $r$ and many $x$. In this section we present an algorithm for the opposite situation, that is for fixed $x$ and many $r$. It is particularly well-suited to computing $K_{i r}(x)$ for values of $r$ that occur unpredictably, using as input a pre-computed table for regularly spaced values of $r$ and the same $x$. This is useful, for example, for computing eigenvalues of the Laplacian on hyperbolic surfaces $[\mathbf{3 8}, \mathbf{4 0}]$.

The main idea is to use a Fourier interpolation. More precisely, suppose $F: \mathbb{R} \rightarrow \mathbb{C}$ is a Schwartz function. Then by a generalization of Shannon's sampling theorem [34], 
for any $r, \theta \in \mathbb{R}, X>0$ and $\ell \in \mathbb{Z}_{\geqslant 0}$, we have

$$
\left|F^{(\ell)}(r)-\frac{d^{\ell}}{d r^{\ell}} \sum_{m \in \theta+\mathbb{Z}} F\left(\frac{m}{X}\right) \operatorname{sinc}(\pi(X r-m))\right| \leqslant 2 \int_{|u| \geqslant \pi X}\left|u^{\ell} \hat{F}(u)\right| d u,
$$

where $\hat{F}(u)=(1 / 2 \pi) \int_{\mathbb{R}} F(r) e^{-i r u} d r$ and $\operatorname{sinc} x=(\sin x) / x$. Note that if $F$ decays rapidly away from some $r_{0} \in \mathbb{R}$ then this gives a rapid method of computing $F^{(\ell)}\left(r_{0}\right)$, provided that we can produce a good estimate for the right-hand side of (30). We will apply this idea to an appropriately weighted version of $\widetilde{K}_{s}(x):=2 \cos (\pi s / 2) K_{s}(x)$.

Let $w$ be the Gaussian $w(r)=e^{-\left(r-r_{0}\right)^{2} / 2 h^{2}}$ for some $h>0, r_{0} \in \mathbb{R}$, and set $F(r):=$ $\widetilde{K}_{i r}(x) w(r)$. By (30), for $\ell \in\{0,1\}$ we have

$$
\begin{aligned}
\left.\frac{\partial^{\ell}}{\partial r^{\ell}} \widetilde{K}_{i r}(x)\right|_{r=r_{0}} & =F^{(\ell)}\left(r_{0}\right) \\
= & \sum_{m \in \theta+\mathbb{Z}} F\left(\frac{m}{X}\right)(\pi X)^{\ell} \operatorname{sinc}^{(\ell)}\left(\pi\left(X r_{0}-m\right)\right)+4 \beta \int_{\pi X}^{\infty} u^{\ell}|\hat{F}(u)| d u \\
= & \sum_{m \in \theta+\mathbb{Z}} \widetilde{K}_{i m / X}(x) \exp \left(-\frac{\left(m / X-r_{0}\right)^{2}}{2 h^{2}}\right)(\pi X)^{\ell} \operatorname{sinc}^{(\ell)}\left(\pi\left(X r_{0}-m\right)\right) \\
& +4 \beta \int_{\pi X}^{\infty} u^{\ell}|\hat{F}(u)| d u
\end{aligned}
$$

with $-1 \leqslant \beta \leqslant 1$, and formulas for higher derivatives may be worked out similarly using the Leibniz rule.

Note that (31) is a convolution, so we can use it together with the fast Fourier transform (FFT) to 'upsample' a course grid of values of $\widetilde{K}_{i r}(x)$ to a finer grid, which can in turn be used for rapid single-point evaluations. Moreover, choosing $\theta=1 / 2$, we can conveniently compute $\widetilde{K}_{i r}(x)$ and $\partial \widetilde{K}_{i r}(x) / \partial r$ for all $r$, including $r=0$.

Our present task is to work out a bound for the error, $4 \int_{\pi X}^{\infty} u^{\ell}|\hat{F}(u)| d u$. We begin with the integral representation

$$
\widetilde{K}_{s}(x)=\int_{-\infty}^{\infty} \cos (x \sinh t) e^{s t} d t
$$

valid for all $s$ with $|\operatorname{Re}(s)|<1$. The integral is only conditionally convergent, but we can improve the convergence by integration by parts. More precisely, if we integrate by parts $n$ times, the result can be expressed in the form

$$
\widetilde{K}_{s}(x)=\int_{-\infty}^{\infty} \frac{\cos ^{(-n)}(x \sinh t)}{(x \cosh t)^{n}} f_{n}(\tanh t, s) e^{s t} d t
$$

where $\cos ^{(-n)}=(-1)^{n} \cos ^{(n)}$ is the $n$th anti-derivative of the cosine function and $f_{n}(\xi, s)$ is a polynomial function of $\xi$ and $s$, defined by the recurrence

$$
f_{0}=1 \quad \text { and } \quad f_{n}=(n \xi-s) f_{n-1}+\left(\xi^{2}-1\right) \frac{\partial f_{n-1}}{\partial \xi} .
$$

From this we see that $f_{n}$ is essentially a Jacobi polynomial,

$$
f_{n}(\xi, s)=n ! P_{n}^{(-s, s)}(\xi)=\sum_{k=0}^{n}\left(\frac{\xi-1}{2}\right)^{k} \frac{(n+k) !}{k !(n-k) !} \prod_{\ell=k+1}^{n}(\ell-s) .
$$

It is also related to the Legendre spherical function $P_{n}^{s}(\xi)$ via

$$
f_{n}(\tanh t, s) e^{s t}=\Gamma(n+1-s) P_{n}^{s}(\tanh t) .
$$


In particular, when $s=0$ we get $n$ ! times the classical Legendre polynomials, $P_{n}(\xi)$, which satisfy the bound $\left|P_{n}(\xi)\right| \leqslant 1=P_{n}(1)$ for $\xi \in[-1,1]$. We conjecture that this can be generalized as follows.

Conjecture 1 . Let $n \in \mathbb{Z}_{\geqslant 0}$. Then

$$
\left|\frac{\partial^{j} f_{n}}{\partial s^{j}}(\xi, i r)\right| \leqslant\left|\frac{\partial^{j} f_{n}}{\partial s^{j}}(1, i r)\right|
$$

for all $\xi \in[-1,1], r \in \mathbb{R}, j \in \mathbb{Z}_{\geqslant 0}$.

We give the following evidence in favor of the conjecture.

Lemma 7. Inequality (34) is true if any of the following holds:

(i) $r=0$;

(ii) $j=0$;

(iii) $n \leqslant j+100$.

In particular, Conjecture 1 is true for all $n \leqslant 101$.

The proof is given in the appendix on page 102 .

REMARK. It is easy to check Conjecture 1 for any given value of $n$, and thus we are free to assume it as long as we include this verification as part of the algorithm for evaluating (31). The key point is that $\left\langle\left|\left(\partial^{j} / \partial s^{j}\right) f_{n}(\cdot, i r)\right|^{2}, P_{k}\right\rangle$ turns out to be non-negative; in fact it has all non-negative coefficients as a polynomial in $r$, which can be verified in every non-trivial case for a given $n$. To see that this implies Conjecture 1 , note that if $\phi:[-1,1] \rightarrow \mathbb{R}$ is any smooth function such that $\left\langle\phi, P_{k}\right\rangle \geqslant 0$ for all $k$ then

$$
|\phi(\xi)|=\left|\sum_{k=0}^{\infty}\left(k+\frac{1}{2}\right)\left\langle\phi, P_{k}\right\rangle P_{k}(\xi)\right| \leqslant \sum_{k=0}^{\infty}\left(k+\frac{1}{2}\right)\left\langle\phi, P_{k}\right\rangle P_{k}(1)=\phi(1) .
$$

Proposition 4. Let $F(r)=\widetilde{K}_{i r}(x) e^{-\left(r-r_{0}\right)^{2} / 2 h^{2}}$ for some $x>0, h>0, r_{0} \in \mathbb{R}$, and assume $n$ is a positive integer for which Conjecture 1 is true. Then for any positive real number $R \geqslant\left|r_{0}\right|$, we have

$$
|\hat{F}(u)| \leqslant(4 n)^{1 / 4} \exp \left[\frac{n(n+1)(2 n+1)}{12 R^{2}}+\frac{1}{2} n^{2}\left(\frac{1}{h}+\frac{h}{R}\right)^{2}+n \log \frac{2 R}{x}-n u\right] .
$$

The proof is given in the appendix on page 104 .

Note that for $\ell \in\{0,1\}, X>0$, this gives the estimate

$$
\begin{aligned}
& 4 \int_{\pi X}^{\infty} u^{\ell}|\hat{F}(u)| d u \leqslant \frac{4 \sqrt{2}}{n^{3 / 4}}\left(\pi X+n^{-1}\right)^{\ell} \\
& \quad \times \exp \left[\frac{n(n+1)(2 n+1)}{12 R^{2}}+\frac{1}{2} n^{2}\left(\frac{1}{h}+\frac{h}{R}\right)^{2}+n \log \frac{2 R}{x}-\pi n X\right] .
\end{aligned}
$$

When $R$ is large compared to $h^{2}$, the right-hand side of the above is smallest for $n \approx$ $h^{2}(\pi X-\log 2 R / x)$, where it is about $\exp \left(-h^{2}(\pi X-\log (2 R / x))^{2} / 2\right)$; this is consistent with the Gaussian decay that $\hat{F}$ would have if $\widetilde{K}_{i r}(x)$ were band-limited with bandwidth $\log (2 R / x)$.

Since the bound is valid for any $R \geqslant\left|r_{0}\right|$, we may estimate the error term in (31) once and for all by taking $R$ to be the largest value of $r_{0}$ that we require. The final ingredient that we will need is a bound for the error incurred by truncating the sum in (31). 
Proposition 5. Let notation be as in Proposition 4. Then for any $\ell \in\{0,1\}, \theta \in \mathbb{R}$ and $X, M \in \mathbb{R}_{>0}$, we have

$$
\begin{gathered}
\sum_{\substack{m \in \theta+\mathbb{Z} \\
\left|m-X r_{0}\right| \geqslant M}}\left|F\left(\frac{m}{X}\right)(\pi X)^{\ell} \operatorname{sinc}^{(\ell)}\left(\pi\left(X r_{0}-m\right)\right)\right| \\
<\frac{16}{\pi M x^{1 / 3}}\left(X \sqrt{\pi^{2}+M^{-2}}\right)^{\ell} \frac{e^{-M^{2} /\left(2 h^{2} X^{2}\right)}}{1-e^{-M /\left(h^{2} X^{2}\right)}} .
\end{gathered}
$$

The proof is given in the appendix on page 106 .

\section{Appendix. Proofs}

Our first task is to prove Lemma 1 . We first prove the following auxiliary result.

Sublemma 1. For all $0 \leqslant \tau \leqslant 1$ and $u>0$ we have

$$
\sqrt{1-\tau^{2}} \sqrt{1-\frac{\tau^{2} u^{2}}{\sinh ^{2} u}} \leqslant 1-\tau^{2}+\frac{1}{6} \tau^{2} u^{2}
$$

Proof. Squaring and expanding, we see that our task is to prove

$$
-36+12 u^{2}+36 \frac{u^{2}}{\sinh ^{2} u}+\tau^{2}\left(36-12 u^{2}+u^{4}-36 \frac{u^{2}}{\sinh ^{2} u}\right) \geqslant 0 .
$$

Clearly this holds for all $0 \leqslant \tau \leqslant 1$ if and only if it holds for both $\tau=0$ and $\tau=1$. However for $\tau=1$ the inequality is trivial, and for $\tau=0$ the claim is equivalent to $\left(u^{2}-3\right) \sinh ^{2} u+$ $3 u^{2} \geqslant 0$ which is easily seen to hold for all $u \geqslant 0$ using repeated differentiation; indeed the fourth derivative of the left-hand side is $8 u\left(8 \sinh u \cosh u+2 u \cosh ^{2} u-u\right)$, which is clearly non-negative for all $u \geqslant 0$, while all the lower order derivatives vanish at $u=0$.

Proof of Lemma 1. Dividing through by $x$ and writing $\tau:=r / x \in(0,1]$, we see that our task is to prove that the function

$$
f(u):=\cosh u \cos v(u)+\tau v(u)-\cos \alpha-\tau \alpha-\frac{1}{2} \sqrt{1-\tau^{2}} u^{2}
$$

is non-negative for all real $u$. Note that $f$ is even and $f(0)=0$; hence it suffices to prove $f^{\prime}(u) \geqslant 0$ for all $u>0$. We compute

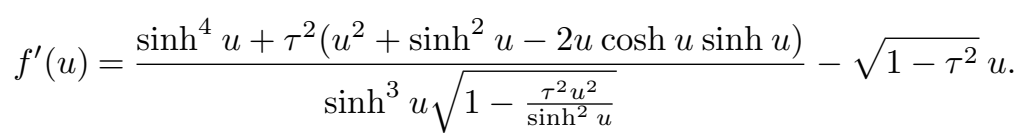

Clearing the denominator and using Sublemma 1, we see that it suffices to prove

$$
\sinh ^{4} u+\tau^{2}\left(u^{2}+\sinh ^{2} u-2 u \cosh u \sinh u\right) \geqslant\left(1-\tau^{2}+\frac{\tau^{2} u^{2}}{6}\right) u \sinh ^{3} u .
$$

Overestimating $\left(1-\tau^{2}\right) u \sinh ^{3} u$ by $\left(1-\tau^{2}\right) \sinh ^{4} u$ and then simplifying, we see that it suffices to prove, for all $u>0$ :

$$
6 \sinh ^{4} u+6 \sinh ^{2} u+6 u^{2}-12 u \cosh u \sinh u-u^{3} \sinh ^{3} u \geqslant 0 .
$$

However this follows by noticing that the left-hand side vanishes at $u=0$, and that its derivative is, for all $u \geqslant 0$ :

$$
\begin{aligned}
& 3(\sinh u)^{2}\left(8 \sinh u \cosh u-u^{3} \cosh u-8 u-u^{2} \sinh u\right) \\
& \quad=3(\sinh u)^{2}\left\{2 \sinh u\left(\cosh u-\frac{1}{2} u^{2}\right)+6 \cosh u\left(\sinh u-\frac{1}{6} u^{3}\right)-8 u\right\} \\
& \quad \geqslant 3(\sinh u)^{2}\{2 \sinh u+6 u \cosh u-8 u\} \geqslant 0
\end{aligned}
$$


Proof of Lemma 2. Let us write $f(u)$ for the difference between the right- and the left-hand side of (11). Arguing as in the proof of Lemma 1 we see that it suffices to prove $f^{\prime}(u) \geqslant 0$ for all $u>0$, namely to prove

$$
\frac{\sinh ^{4} u+\tau^{2}\left(u^{2}+\sinh ^{2} u-2 u \cosh u \sinh u\right)}{\sinh ^{3} u \sqrt{1-\frac{\tau^{2} u^{2}}{\sinh ^{2} u}}} \geqslant \frac{4 \sqrt{3}}{9} \tau u^{2} .
$$

Here we again write $\tau:=r / x \in(0,1]$. Clearing the denominator and squaring, we see that it suffices to prove $\tau^{4} a(u)+\tau^{2} b(u)+c(u) \geqslant 0$ for all $u>0$, where

$$
\begin{aligned}
& a(u)=\left(u^{2}+\sinh ^{2} u-2 u \cosh u \sinh u\right)^{2}+\frac{16}{27} u^{6} \sinh ^{4} u ; \\
& b(u)=2(\sinh u)^{4}\left(u^{2}+\sinh ^{2} u-2 u \cosh u \sinh u\right)-\frac{16}{27} u^{4} \sinh ^{6} u \\
& c(u)=\sinh ^{8} u .
\end{aligned}
$$

Using Taylor expansions and interval arithmetic one checks that

$$
a(u)+b(u)+c(u)>0 \text { and } 2 a(u)+b(u)<0,
$$

for all $u>0$ (cf. [7]; for this and all later verifications using interval arithmetic, we used the intpakX Maple package [17]). For fixed $u>0$, the second inequality in (A.1) together with the obvious fact that $a(u)>0$ imply that the function $\tau \mapsto \tau^{4} a(u)+\tau^{2} b(u)+c(u)$ is decreasing for $\tau \in[0,1]$. In particular this function takes its minimum at $\tau=1$, and using also the first inequality in (A.1) we conclude $\tau^{4} a(u)+\tau^{2} b(u)+c(u)>0$, as desired.

Proof of Lemma 3. We have

$$
v^{\prime}(u)=\frac{\tau(\sinh u-u \cosh u)}{(\sinh u)^{2} \sqrt{1-\frac{\tau^{2} u^{2}}{\sinh ^{2} u}}}=\frac{\sinh u-u \cosh u}{(\sinh u) \sqrt{\tau^{-2} \sinh ^{2} u-u^{2}}},
$$

where, again, $\tau:=r / x \in(0,1]$. Hence $v^{\prime}(u)<0$ for all $u>0$, since $\sinh u-u \cosh u<0$ for these $u$. Using $\tau^{-2} \sinh ^{2} u \geqslant \sinh ^{2} u \geqslant u^{2}+\frac{1}{3} u^{4}$ it also follows that

$$
-v^{\prime}(u) \leqslant \sqrt{3} \frac{u \cosh u-\sinh u}{u^{2} \sinh u}=\sqrt{3} \frac{\sum_{m=1}^{\infty} \frac{2 m}{(2 m+1) !} u^{2 m+1}}{\sum_{m=1}^{\infty} \frac{1}{(2 m-1) !} u^{2 m+1}}<3^{-\frac{1}{2}}
$$

since

$$
\frac{2 m}{(2 m+1) !}\left(\frac{1}{(2 m-1) !}\right)^{-1}=\frac{1}{2 m+1} \leqslant \frac{1}{3} \quad \text { for all } m \geqslant 1,
$$

with strict inequality for $m \geqslant 2$. Hence (12) is proved.

Using the first relation in (A.2) we also get

$$
\left|u v^{\prime}(u)\right| \leqslant \sqrt{3}\left(\operatorname{coth} u-u^{-1}\right) .
$$

Here the right-hand side is strictly increasing for all $u>0$, and has limit $\sqrt{3}$ as $u \rightarrow \infty$. Hence also (13) holds.

Proof of Proposition 1. First of all, using (4) and Lemmata 1 and 2 we have

$$
0<K_{i r}(x) \leqslant e^{-x \cos \alpha-r \alpha} \min \left(\int_{0}^{\infty} e^{-\frac{1}{2} \sqrt{x^{2}-r^{2}} u^{2}} d u, \int_{0}^{\infty} e^{-\frac{4 \sqrt{3}}{27} r u^{3}} d u\right) .
$$

Evaluating the two integrals we obtain (14).

We next prove (17). Thus assume $j_{1}, j_{2} \in \mathbb{Z}_{\geqslant 0}, \varepsilon>0$ and $r>0, x \geqslant \max (\varepsilon, r)$. By differentiating under the integration sign in (1) and then moving to the path of steepest descent, we obtain

$$
\frac{\partial^{j_{1}+j_{2}}}{\partial r^{j_{1}} \partial x^{j_{2}}} K_{i r}(x)=\operatorname{Re} \int_{0}^{\infty}(i u-v(u))^{j_{1}}(-\cosh (u+i v(u)))^{j_{2}} e^{\eta(u)}\left(1+i v^{\prime}(u)\right) d u .
$$


We continue to write $\tau:=r / x \in(0,1]$. Using Lemma 3 and $\cosh (i v(0))=\sqrt{1-\tau^{2}}$ we see that $\left|\cosh (u+i v(u))-\sqrt{1-\tau^{2}}\right| \ll u$ for all $u \in[0,1]$. On the other hand for $u \geqslant 1$ we use $|\cosh (u+i v(u))| \leqslant e^{u}$. It follows that, again using Lemma 3 ,

$$
\left|\frac{\partial^{j_{1}+j_{2}}}{\partial r^{j_{1}} \partial x^{j_{2}}} K_{i r}(x)\right| \ll \int_{0}^{1}\left(\sqrt{1-\tau^{2}}+u\right)^{j_{2}} e^{\eta(u)} d u+\int_{1}^{\infty} u^{j_{1}} e^{j_{2} u+\eta(u)} d u .
$$

Let us first assume $x \geqslant r+r^{\frac{1}{3}}$. Then since also $x \geqslant \varepsilon$ we have $x-r \gg_{\varepsilon} x^{1 / 3}$ and $x^{2}-r^{2} \gg_{\varepsilon}$ $x^{4 / 3} \gg_{\varepsilon} 1$. We now get, using Lemma 1 ,

$$
\begin{aligned}
& \left|\frac{\partial^{j_{1}+j_{2}}}{\partial r^{j_{1}} \partial x^{j_{2}}} K_{i r}(x)\right| \ll e^{-(\pi / 2) r} e^{-\sqrt{x^{2}-r^{2}}+r \arccos (r / x)} \\
& \quad \quad \times\left\{\int_{0}^{\infty}\left(\left(1-\tau^{2}\right)^{j_{2} / 2}+u^{j_{2}}\right) e^{-\frac{1}{2} \sqrt{x^{2}-r^{2}} u^{2}} d u+\int_{1}^{\infty} e^{\left(j_{2}+1\right) u-\frac{1}{2} \sqrt{x^{2}-r^{2}} u^{2}} d u\right\} \\
& \quad \ll e^{-(\pi / 2) r} e^{-\sqrt{x^{2}-r^{2}}+r \arccos (r / x)}\left\{\left(x^{2}-r^{2}\right)^{j_{2} / 2-\frac{1}{4}} x^{-j_{2}}+\left(x^{2}-r^{2}\right)^{-\left(j_{2}+1\right) / 4}\right\} \\
& \quad \ll e^{-(\pi / 2) r} e^{-\sqrt{x^{2}-r^{2}}+r \arccos (r / x)}\left(x^{2}-r^{2}\right)^{j_{2} / 2-\frac{1}{4}} x^{-j_{2}} .
\end{aligned}
$$

(Recall that we allow the implied constant to depend on $j_{1}, j_{2}, \varepsilon$ only.) In the remaining case, $r \leqslant x<r+r^{\frac{1}{3}}$, we necessarily have $r \gg_{\varepsilon} 1$ because of $x \geqslant \varepsilon$, and we now get, using Lemma 2 and writing $c=4 \sqrt{3} / 27$,

$$
\begin{aligned}
& \left|\frac{\partial^{j_{1}+j_{2}}}{\partial r^{j_{1}} \partial x^{j_{2}}} K_{i r}(x)\right| \ll e^{-(\pi / 2) r} e^{-\sqrt{x^{2}-r^{2}}+r \arccos (r / x)} \\
& \quad \quad \times\left\{\int_{0}^{\infty}\left(\left(1-\tau^{2}\right)^{j_{2} / 2}+u^{j_{2}}\right) e^{-c r u^{3}} d u+\int_{1}^{\infty} e^{\left(j_{2}+1\right) u-c r u^{3}} d u\right\} \\
& \quad \ll e^{-(\pi / 2) r} e^{-\sqrt{x^{2}-r^{2}}+r \arccos (r / x)}\left\{\left(x^{2}-r^{2}\right)^{j_{2} / 2} x^{-j_{2}} r^{-\frac{1}{3}}+r^{-\left(j_{2}+1\right) / 3}\right\} \\
& \quad \ll e^{-(\pi / 2) r} e^{-\sqrt{x^{2}-r^{2}}+r \arccos (r / x)} r^{-\left(j_{2}+1\right) / 3} .
\end{aligned}
$$

Noticing also that $x<r+r^{\frac{1}{3}}$ implies $x \ll_{\varepsilon} r$ we have now completed the proof of (17).

Finally we prove (15) and (16). By (A.4) we have

$$
\frac{\partial}{\partial r} K_{i r}(x)=-\int_{0}^{\infty}\left(u v^{\prime}(u)+v(u)\right) e^{\eta(u)} d u
$$

and

$$
\frac{\partial^{2}}{\partial r^{2}} K_{i r}(x)=\int_{0}^{\infty}\left(v(u)^{2}+2 u v(u) v^{\prime}(u)-u^{2}\right) e^{\eta(u)} d u
$$

Note that $-\sqrt{3}<u v^{\prime}(u)+v(u)<\pi / 2$ for all $u>0$, because of $0<v(u)<\pi / 2$ and Lemma 3 . Hence, using Lemmata 1 and 2 , we see that $\left|(\partial / \partial r) K_{i r}(x)\right|$ is bounded from above by $\sqrt{3}$ times the right-hand side in (A.3). We thus obtain (15). Similarly, for all $u>0$ we have $v(u)^{2}+$ $2 u v(u) v^{\prime}(u)<v(u)^{2}<\pi^{2} / 4$ and $v(u)^{2}+2 u v(u) v^{\prime}(u)>v(u)^{2}-2 \sqrt{3} v(u)>\pi(\pi / 4-\sqrt{3})$, and hence by Lemmata 1 and 2 ,

$$
\begin{aligned}
&\left|\frac{\partial^{2}}{\partial r^{2}} K_{i r}(x)\right| \leqslant e^{-x \cos \alpha-r \alpha} \min \left(\int_{0}^{\infty}\left(\pi\left(\sqrt{3}-\frac{\pi}{4}\right)+u^{2}\right) e^{-\frac{1}{2} \sqrt{x^{2}-r^{2}} u^{2}} d u,\right. \\
&\left.\int_{0}^{\infty}\left(\pi\left(\sqrt{3}-\frac{\pi}{4}\right)+u^{2}\right) e^{-\frac{4 \sqrt{3}}{27} r u^{3}} d u\right) .
\end{aligned}
$$

Evaluating the two integrals we obtain (16).

We next turn to the proof of Lemma 4. Unfortunately we have not been able to find an elegant proof of this result; our proof is lengthy (it goes from here to page 97), it splits into several cases, and at several steps we make use of (rigorous) machine computations. 
Proof of Lemma 4, the inequality (19). After dividing through by $x$, our task is to prove that for any $\mu>0$ and any $u \geqslant u_{\pi}$, we have

$$
\cosh u \cos v+(\cosh \mu)\left(v-\frac{\pi}{2}\right) \geqslant(u-\mu)^{2} \sinh \mu .
$$

Case I: Assume $0<\mu \leqslant u$. Then $v \in(0, \pi / 2]$; thus $\pi / 2-v=\arcsin (\cos v)$. Hence, writing $h:=u-\mu \geqslant 0$, our task is to prove that for all $\mu>0$ and $h \geqslant 0$,

$$
(\cosh (\mu+h)) A^{\frac{1}{2}}-(\cosh \mu) \arcsin \left(A^{\frac{1}{2}}\right) \geqslant h^{2} \sinh \mu,
$$

where $A=A(\mu, h)$ is given by

$$
A(\mu, h)=\cos ^{2} v=1-\frac{(T u-S)^{2}}{\sinh ^{2} u}=1-\frac{(\sinh \mu+h \cosh \mu)^{2}}{\sinh ^{2}(\mu+h)} .
$$

It is natural to also set $A(0,0):=0$; then $A(\mu, h)$ is a continuous function of $(\mu, h) \in\left(\mathbb{R}_{\geqslant 0}\right)^{2}$, and $0 \leqslant A(\mu, h) \leqslant 1$ everywhere. We will repeatedly need the following facts.

Sublemma 2. $A(\mu, h)$ is an increasing function of $\mu \geqslant 0$ for any fixed $h \geqslant 0$, and an increasing function of $h \geqslant 0$ for any fixed $\mu \geqslant 0$. We have $\lim _{\mu \rightarrow \infty} A(\mu, h)=1-(1+h)^{2} e^{-2 h}$ for any fixed $h \geqslant 0$.

Proof. Immediate by differentiation and direct computation.

Case I, Step 1: Proof of (A.5) when $0 \leqslant h \leqslant 0.3$. One checks that $\arcsin (x) \leqslant x+\frac{1}{6} x^{3}+\frac{1}{2} x^{5}$ for all $x \in[0,1]$ (cf. [7]). Furthermore for $0 \leqslant h \leqslant 0.7$ we have $\sqrt{1-(1+h)^{2} e^{-2 h}} \leqslant h-\frac{1}{3} h^{2}$ (cf. [7]), and hence $A(\mu, h)^{\frac{1}{2}} \leqslant h-\frac{1}{3} h^{2}$ for all $\mu \geqslant 0$ (cf. Sublemma 2). Hence for $\mu \geqslant 0$ and $0 \leqslant h \leqslant 0.7$, (A.5) will follow if we can prove

$$
h^{2} \sinh \mu+\frac{1}{6}\left(h-\frac{1}{3} h^{2}\right)^{3} \cosh \mu+\frac{1}{2}\left(h-\frac{1}{3} h^{2}\right)^{5} \cosh \mu \leqslant(\cosh (\mu+h)-\cosh u) A^{\frac{1}{2}} .
$$

Next note that, for all $h \in \mathbb{R}$,

$$
\left(h-\frac{1}{3} h^{2}\right)^{5}-\left(h^{5}-\frac{5}{3} h^{6}+\frac{10}{9} h^{7}\right)=-3^{-5} h^{8}\left(\left(h-\frac{15}{2}\right)^{2}+\frac{135}{4}\right) \leqslant 0 .
$$

Furthermore, by the Taylor expansion of $h \mapsto \cosh (\mu+h)$ we have

$$
\cosh (\mu+h)-\cosh \mu \geqslant h \sinh \mu+\frac{1}{2} h^{2} \cosh \mu+\frac{1}{6} h^{3} \sinh \mu,
$$

by the definition of $A$ and the Taylor expansion of $h \mapsto \sinh ^{2}(\mu+h)$ we have

$$
\begin{aligned}
(\sinh (\mu+h))^{2} A \geqslant & h^{2} \sinh ^{2} \mu+\frac{4}{3} h^{3} \sinh \mu \cosh \mu+\frac{1}{3} h^{4}\left(2 \sinh ^{2} \mu+1\right) \\
& +\frac{4}{15} h^{5} \sinh \mu \cosh \mu+\frac{2}{45} h^{6}\left(2 \sinh ^{2} \mu+1\right) .
\end{aligned}
$$

Furthermore, we have

$$
\sinh (\mu+h) \leqslant \sinh \mu+h \cosh \mu+\frac{1}{2} h^{2} \sinh \mu+\frac{1}{5} h^{3} \cosh \mu, \forall \mu \geqslant 0, h \in[0,0.6] .
$$

This is proved in [7], by verifying that if $f(\mu, h)$ denotes the difference between the right- and the left-hand side of (A.8) then $f \geqslant(\partial / \partial \mu) f$ for all $\mu, h \geqslant 0$, and $\lim _{\mu \rightarrow \infty} e^{-\mu} f(\mu, h)>0$ for all $h \in(0,0.6]$.

Using the above facts, we see that for $\mu \geqslant 0$ and $0 \leqslant h \leqslant 0.6$, (A.7) will follow if we can prove

$$
\begin{aligned}
\left(h^{2} \sinh \mu+\frac{1}{6}\left(h-\frac{1}{3} h^{2}\right)^{3} \cosh \mu+\frac{1}{2}\left(h^{5}-\frac{5}{3} h^{6}+\frac{10}{9} h^{7}\right) \cosh \mu\right)^{2} \\
\quad \times\left(\sinh \mu+h \cosh \mu+\frac{1}{2} h^{2} \sinh \mu+\frac{1}{5} h^{3} \cosh \mu\right)^{2} \\
\leqslant\left(h \sinh \mu+\frac{1}{2} h^{2} \cosh \mu+\frac{1}{6} h^{3} \sinh \mu\right)^{2}\left(h^{2} \sinh ^{2} \mu+\frac{4}{3} h^{3} \sinh \mu \cosh \mu\right. \\
\left.\quad+\frac{1}{3} h^{4}\left(2 \sinh ^{2} \mu+1\right)+\frac{4}{15} h^{5} \sinh \mu \cosh \mu+\frac{2}{45} h^{6}\left(2 \sinh ^{2} \mu+1\right)\right) .
\end{aligned}
$$


The difference between the right- and the left-hand side in (A.9) is clearly a polynomial of degree 20 in $h$, say $\sum_{j=0}^{20} c_{j}(\mu) h^{j}$, where each $c_{j}(\mu)$ is a rational linear combination of $e^{4 \mu}, e^{2 \mu}, 1, e^{-2 \mu}, e^{-4 \mu}$. In fact it turns out that $c_{0}, c_{1}, c_{2}, c_{3}, c_{4}, c_{5}$ are identically zero, and $c_{6}(0)=c_{7}(0)=0$ while $c_{8}(0)=\frac{1}{18}$ (cf. [7]). In fact

$$
c_{6}(\mu)=\frac{1}{72} e^{-4 \mu}\left(e^{2 \mu}-1\right)^{2}\left(\left(e^{2 \mu}-1\right)^{2}+7\left(e^{2 \mu}-1\right)+4\right)
$$

and

$$
c_{7}(\mu)=-\frac{57}{1440} e^{-4 \mu}\left(e^{2 \mu}-1\right)\left(e^{2 \mu}+1\right)\left(e^{2 \mu}+\frac{5 \sqrt{757}-172}{57}\right)\left(e^{2 \mu}-\frac{172+5 \sqrt{757}}{57}\right)
$$

(cf. [7]), from which we see that $c_{6}(\mu) \geqslant 0$ for all $\mu \geqslant 0$ and (noticing also $(5 \sqrt{757}-172) / 57>$ $(5 \cdot 23-172) / 57=-1)$ that $c_{7}(\mu) \geqslant 0$ for all $0 \leqslant \mu \leqslant \frac{1}{2} \log ((172+5 \sqrt{757}) / 57)=0.84606 \ldots$ In particular for $0 \leqslant \mu \leqslant 0.8$ and $h \geqslant 0$ it follows that (A.9) will follow if we can prove

$$
\sum_{j=0}^{12} c_{j+8}(\mu) h^{j} \geqslant 0 .
$$

Using interval arithmetic this inequality is verified to hold, with strict inequality, for all $\langle\mu, h\rangle \in[0,0.2] \times[0,0.35]$, cf. [7]. (This computation is quite quick: the positivity is obtained by computing $\sum_{j=0}^{12} c_{j+8}(\mu) h^{j}$ in interval arithmetic for just ten boxes of the form $\left[U_{j}, U_{j+1}\right] \times$ [0, 0.35], $0=U_{1}<U_{2}<\ldots<U_{11}=0.2$.)

Also using interval arithmetic, $\sum_{j=0}^{14} c_{j+6}(\mu) h^{j}>0$ is verified to hold for all $\langle\mu, h\rangle \in[0.2,2] \times$ $[0,0.35]$ and all $\langle\mu, h\rangle \in[2, \infty) \times[0,0.3]$, cf. [7]. (In fact we first divide through by $e^{4 \mu}$, that is we actually verify that $\sum_{j=0}^{14}\left(e^{-4 \mu} c_{j+6}(\mu)\right) h^{j}>0$; the point is that each $e^{-4 \mu} c_{k}(\mu)$ can be bounded from above and below also for $\mu$ in intervals extending to $\infty$.)

This concludes the proof that (A.5) holds whenever $0 \leqslant h \leqslant 0.3$. (And in fact we have also proved that (A.5) holds whenever $0 \leqslant \mu \leqslant 2$ and $0 \leqslant h \leqslant 0.35$.)

Case I, Step 2: Proof of (A.5) when $h \geqslant 3$. For any $\mu \geqslant 0$ and $h \geqslant 3$ we have

$$
A(\mu, h) \geqslant A(0,3)=1-\frac{3^{2}}{\sinh ^{2} 3}=0.910 \ldots>\frac{9}{10},
$$

by Sublemma 2 . Hence also $A(\mu, h)^{\frac{1}{2}}>\frac{9}{10}$, and

$$
\begin{aligned}
& \cosh (\mu+h) A^{\frac{1}{2}}-(\cosh \mu) \arcsin \left(A^{\frac{1}{2}}\right)-h^{2} \sinh \mu \\
& \quad>\frac{9}{10} \cosh (\mu+h)-\frac{\pi}{2} \cosh \mu-h^{2} \sinh \mu \\
& \quad=\left(\frac{9}{10} \cosh h-\frac{\pi}{2}\right) \cosh \mu+\left(\frac{9}{10} \sinh h-h^{2}\right) \sinh \mu .
\end{aligned}
$$

However, one checks that $\frac{9}{10} \cosh h>\pi / 2$ and $\frac{9}{10} \sinh h>h^{2}$ for all $h \geqslant 3$; hence the above expression is positive and we have proved that (A.5) holds whenever $h \geqslant 3$.

Case I, Step 3: Proof of (A.5) when $\mu \geqslant 2$ and $0.3 \leqslant h \leqslant 3$.

Sublemma 3. For any fixed $\mu, h \geqslant 0$, the function

$$
x \mapsto(\cosh (\mu+h)) x-(\cosh \mu) \arcsin x
$$

is increasing for $0 \leqslant x \leqslant\left(1-\left(\cosh ^{2} \mu\right) / \cosh ^{2}(\mu+h)\right)^{\frac{1}{2}}$ and decreasing for $\left(1-\left(\cosh ^{2} \mu\right) / \cosh ^{2}(\mu+\right.$ $h))^{\frac{1}{2}} \leqslant x \leqslant 1$. In particular the function is increasing for $0 \leqslant x \leqslant A(\mu, h)^{\frac{1}{2}}$.

Proof. The statement in the first sentence is immediate by differentiation. Now to prove the last statement we only have to check that $A(\mu, h)^{\frac{1}{2}} \leqslant\left(1-\left(\cosh ^{2} \mu\right) / \cosh ^{2}(\mu+h)\right)^{\frac{1}{2}}$. 
A sufficient condition for this is, by Sublemma 2: $1-(1+h)^{2} e^{-2 h} \leqslant 1-\left(\cosh ^{2} \mu\right) / \cosh ^{2}$ $(\mu+h)$. This inequality is verified to hold using $\cosh (\mu+h)=\cosh \mu \cosh h+\sinh \mu \sinh h \geqslant$ $\cosh \mu \cosh h$ and $(1+h) \cosh h-e^{h}=h \cosh h-\sinh h \geqslant 0$.

Sublemma 4. If $0 \leqslant h_{0}<h_{1}$ and $U>0$ are any numbers such that the quantity

$$
\begin{aligned}
M\left(h_{0}, h_{1}, U\right):= & \sqrt{A\left(U, h_{0}\right)} e^{h_{0}}-\arcsin \sqrt{A\left(U, h_{0}\right)}-h_{1}^{2} \\
& -(1-\tanh U) \max \left\{0, \sqrt{A\left(U, h_{0}\right)} \sinh h_{1}-h_{0}^{2}\right\}
\end{aligned}
$$

is non-negative, then the inequality (A.5) holds for all $\mu, h$ satisfying $\mu \geqslant U$ and $h \in\left[h_{0}, h_{1}\right]$.

Proof. Assume that $0 \leqslant h_{0}<h_{1}$ and $U>0$ satisfy $M\left(h_{0}, h_{1}, U\right) \geqslant 0$, and fix arbitrary numbers $\mu, h$ satisfying $\mu \geqslant U$ and $h \in\left[h_{0}, h_{1}\right]$. By Sublemma 2 we have $A\left(U, h_{0}\right) \leqslant A(\mu, h)$. Hence by Sublemma 3,

$$
\begin{aligned}
& (\cosh (\mu+h)) A(\mu, h)^{\frac{1}{2}}-(\cosh \mu) \arcsin \left(A(\mu, h)^{\frac{1}{2}}\right) \\
& \quad \geqslant(\cosh (\mu+h)) A\left(U, h_{0}\right)^{\frac{1}{2}}-(\cosh \mu) \arcsin \left(A\left(U, h_{0}\right)^{\frac{1}{2}}\right),
\end{aligned}
$$

and to prove (A.5) for our $\mu, h$ it now suffices to prove that the right-hand side of (A.10) is at least $h^{2} \sinh \mu$, or equivalently to prove

$$
A_{0}^{\frac{1}{2}} \cosh h-\arcsin \left(A_{0}^{\frac{1}{2}}\right) \geqslant\left(h^{2}-A_{0}^{\frac{1}{2}} \sinh h\right) \tanh \mu,
$$

where $A_{0}:=A\left(U, h_{0}\right)$. But $\tanh U \leqslant \tanh \mu<1$, and hence the following inequality implies (A.11):

$$
A_{0}^{\frac{1}{2}} \cosh h-\arcsin \left(A_{0}^{\frac{1}{2}}\right) \geqslant h^{2}-A_{0}^{\frac{1}{2}} \sinh h+(1-\tanh U) \max \left\{0, A_{0}^{\frac{1}{2}} \sinh h-h^{2}\right\} .
$$

Using $A_{0}^{\frac{1}{2}}(\cosh h+\sinh h)=A_{0}^{\frac{1}{2}} e^{h}$ and $h \in\left[h_{0}, h_{1}\right] \subset \mathbb{R}_{\geqslant 0}$, we see that (A.12) follows from our assumption $M\left(h_{0}, h_{1}, U\right) \geqslant 0$.

In [7] we check that there is a sequence $0.3=h_{1}<h_{2}<\ldots<h_{n}=3$ such that $M\left(h_{j}, h_{j+1}, 2\right)>0$ for each $j \in\{1,2, \ldots, n-1\}$. (In fact the sequence which we find in [7] has $n=199$ and smallest step size $\min _{j}\left(h_{j+1}-h_{j}\right)=2^{-8} \cdot 5^{-1}$.) Hence, in view of Sublemma 4, we have now proved that the inequality (A.5) holds for all $\langle\mu, h\rangle$ satisfying $\mu \geqslant 2$ and $0.3 \leqslant h \leqslant 3$.

In fact, we also check in [7] that there is a sequence $0.35=h_{1}<h_{2}<\ldots<h_{n}=3$ (with $n=273)$ such that $M\left(h_{j}, h_{j+1}, 1.3\right)>0$ for each $j \in\{1,2, \ldots, n-1\}$. Hence we also have: the inequality (A.5) holds for all $(\mu, h)$ satisfying $\mu \geqslant 1.3$ and $0.35 \leqslant h \leqslant 3$.

Case I, Step 4: Proof of (A.5) when $0<\mu \leqslant 2$ and $0.3 \leqslant h \leqslant 3$. We do this in [7] using brute force interval arithmetic. In fact we prove that (A.5) holds, with strict inequality, for all $\langle\mu, h\rangle \in[0,2] \times[0.3,3]$, by splitting this box into several smaller boxes, and computing the interval arithmetic version of the difference of the two sides in (A.5) for each such small box.

To calculate the difference of the two sides in (A.5) reasonably efficiently in interval arithmetic we make strong use of the monotonicity properties recorded in Sublemmas 2 and 3.

The computation in [7] to prove the above claim takes about 32 minutes on a $2.2 \mathrm{GHz} \mathrm{PC}$. The successful splitting of [0,2] $\times[0.3,3]$ found in [7] consists of 292530 boxes, the majority of which have size $2^{-9} 5^{-1} \times 2^{-9} 5^{-1}$.

Note that steps $1-4$ together prove that (A.5) holds for all $\mu, h \geqslant 0$.

We remark that a considerable amount of computer time may be saved by recalling that in step 1 we also proved (A.5) for all $\langle\mu, h\rangle \in[0,2] \times[0,0.35]$, and in step 3 we also proved (A.5) for all $\langle\mu, h\rangle \in[1.3, \infty) \times[0.35,3]$. Hence in step 4 it actually suffices to prove that (A.5) holds for all $\langle\mu, h\rangle \in[0,1.3] \times[0.35,3]$. Using brute force interval arithmetic as before this only takes about 4 minutes on a $2.2 \mathrm{GHz} \mathrm{PC}$, using a splitting of $[0,1.3] \times[0.35,3]$ into 38721 boxes, cf. [7]. 
Case II: Assume $u_{\pi} \leqslant u \leqslant \mu$. Then $v \in[\pi / 2, \pi]$ and thus $\cos v \leqslant 0$ and $\pi / 2-v=$ $\arcsin (\cos v)$. Hence, writing $h:=\mu-u$, our task is to prove that for all $\mu>0$ and $h \in$ $[0, \tanh \mu]$,

$$
-(\cosh (\mu-h)) B^{\frac{1}{2}}+(\cosh \mu) \arcsin \left(B^{\frac{1}{2}}\right) \geqslant h^{2} \sinh \mu,
$$

where

$$
B=B(\mu, h)=\cos ^{2} v=1-\frac{(\sinh \mu-h \cosh \mu)^{2}}{\sinh ^{2}(\mu-h)} .
$$

Note that $\sinh \mu-h \cosh \mu \geqslant 0$ and $0 \leqslant B(\mu, h) \leqslant 1$ for all $\mu>0$ and $h \in[0, \tanh \mu]$.

Sublemma 5. For fixed $\mu>0, B(\mu, h)$ is an increasing function of $h \in[0, \tanh \mu]$. For fixed $0 \leqslant h<1, B(\mu, h)$ is a decreasing function of $\mu \in[\operatorname{artanh} h, \infty) \cap \mathbb{R}_{>0}$ satisfying $\lim _{\mu \rightarrow \infty} B(\mu, h)=1-(1-h)^{2} e^{2 h}$.

Proof. Again immediate by differentiation and direct computation.

Case II, Step 1: Proof of (A.13) when $[0<\mu \leqslant 0.58$ and $0 \leqslant h \leqslant \tanh \mu]$ or $[\mu \geqslant 0.58$ and $0 \leqslant$ $h \leqslant 0.5]$. One checks that $\arcsin (x) \geqslant x+\frac{1}{6} x^{3}+\frac{3}{40} x^{5}$ for all $x \in[0,1]$ (cf. [7]). Furthermore for $0 \leqslant h \leqslant 0.55$ we have $\sqrt{1-(1-h)^{2} e^{2 h}} \geqslant h+\frac{1}{4} h^{2}$ (again cf. [7]), and hence $B(\mu, h)^{\frac{1}{2}} \geqslant h+\frac{1}{4} h^{2}$ for all $\mu \geqslant \operatorname{artanh} h(\mu>0)$, by Sublemma 5 . Hence for any $\mu>0$ and $0 \leqslant h \leqslant \min (0.55, \tanh \mu)$, (A.13) will follow if we can prove

$$
h^{2} \sinh \mu \leqslant(\cosh \mu-\cosh (\mu-h)) B^{\frac{1}{2}}+\frac{1}{6}\left(h+\frac{1}{4} h^{2}\right)^{3} \cosh \mu+\frac{3}{40} h^{5} \cosh \mu .
$$

Next, from the Taylor expansion of $h \mapsto \cosh (\mu-h)$ we see that, for any $0 \leqslant h \leqslant \mu$,

$$
\cosh \mu-\cosh (\mu-h) \geqslant h \sinh \mu-\frac{1}{2} h^{2} \cosh \mu+\frac{1}{6} h^{3} \sinh \mu-\frac{1}{24} h^{4} \cosh \mu .
$$

Note that the right-hand side in this inequality is certainly non-negative whenever $0 \leqslant h \leqslant$ tanh $\mu$, since then $\frac{1}{2} h^{2} \cosh \mu \leqslant \frac{1}{2} h \sinh \mu$ and $\frac{1}{24} h^{4} \cosh \mu \leqslant \frac{1}{24} h^{3} \sinh \mu$. Hence, by squaring and using the definition of $B(\mu, h)$, we see that (A.14) will follow if we can prove

$$
\begin{aligned}
& \left(h^{2} \sinh \mu-\frac{1}{6}\left(h+\frac{1}{4} h^{2}\right)^{3} \cosh \mu-\frac{3}{40} h^{5} \cosh \mu\right)^{2} \sinh ^{2}(\mu-h) \\
& \leqslant\left(h \sinh \mu-\frac{1}{2} h^{2} \cosh \mu+\frac{1}{6} h^{3} \sinh \mu-\frac{1}{24} h^{4} \cosh \mu\right)^{2} \\
& \quad \times\left(\sinh ^{2}(\mu-h)-(\sinh \mu-h \cosh \mu)^{2}\right) .
\end{aligned}
$$

Next, from the Taylor expansion of $h \mapsto \sinh ^{2}(\mu-h)$ we see that, for any $0 \leqslant h \leqslant \mu$, $0 \leqslant \sinh ^{2}(\mu-h)-\left\{\sinh ^{2} \mu-2 h \sinh \mu \cosh \mu+h^{2}\left(2 \sinh ^{2} \mu+1\right)-\frac{4}{3} h^{3} \sinh \mu \cosh \mu\right.$

$$
\left.+\frac{1}{3} h^{4}\left(2 \sinh ^{2} \mu+1\right)-\frac{4}{15} h^{5} \sinh \mu \cosh \mu\right\} \leqslant \frac{2}{45} h^{6}\left(2 \sinh ^{2} \mu+1\right) .
$$

Hence we conclude that, for any $\mu>0$ and $0 \leqslant h \leqslant \min (0.55, \tanh \mu),($ A.13) will follow if we can prove

$$
\begin{aligned}
&\left\{h^{2} \sinh \mu-\frac{1}{6}\left(h+\frac{1}{4} h^{2}\right)^{3} \cosh \mu-\frac{3}{40} h^{5} \cosh \mu\right\}^{2} \\
& \times\left\{\sinh ^{2} \mu-2 h \sinh \mu \cosh \mu+h^{2}\left(2 \sinh ^{2} \mu+1\right)-\frac{4}{3} h^{3} \sinh \mu \cosh \mu\right. \\
&\left.\quad+\frac{1}{3} h^{4}\left(2 \sinh ^{2} \mu+1\right)-\frac{4}{15} h^{5} \sinh \mu \cosh \mu+\frac{2}{45} h^{6}\left(2 \sinh ^{2} \mu+1\right)\right\} \\
& \leqslant\left\{h \sinh \mu-\frac{1}{2} h^{2} \cosh \mu+\frac{1}{6} h^{3} \sinh \mu-\frac{1}{24} h^{4} \cosh \mu\right\}^{2} \\
& \times\left\{h^{2} \sinh ^{2} \mu-\frac{4}{3} h^{3} \sinh \mu \cosh \mu+\frac{1}{3} h^{4}\left(2 \sinh ^{2} \mu+1\right)\right. \\
&\left.\quad-\frac{4}{15} h^{5} \sinh \mu \cosh \mu\right\} .
\end{aligned}
$$

The difference between the right- and the left-hand side in (A.16) is clearly a polynomial of degree 18 in $h$, say $\sum_{j=0}^{18} c_{j}(\mu) h^{j}$, where each $c_{j}(\mu)$ is a rational linear combination of $e^{4 \mu}, e^{2 \mu}, 1, e^{-2 \mu}, e^{-4 \mu}$. In fact it turns out that $c_{0}, c_{1}, c_{2}, c_{3}, c_{4}, c_{5}$ are identically zero, 
and $c_{6}(0)=c_{7}(0)=0$ while $c_{8}(0)=\frac{1}{18} \quad($ cf. [7]). In particular (A.16) is equivalent with $f(\mu, h) \geqslant 0$, where

$$
f(\mu, h):=\sum_{j=0}^{12} c_{j+6}(\mu) h^{j} .
$$

Now using interval arithmetic one proves that $\left(\partial^{2} / \partial h^{2}\right) f(\mu, h)>0$ whenever $0 \leqslant h \leqslant$ $\mu \leqslant 0.6$, and also, if $g(\mu, h):=(\partial / \partial h) f(\mu, h)$ then $(d / d h) g(h, h)<0$ for all $h \in[0,0.6]$ (cf. [7]). Since $g(0,0)=c_{7}(0)=0$, it follows that $g(h, h) \leqslant 0$ for all $h \in[0,0.6]$, and also $g(\mu, h)=$ $(\partial / \partial h) f(\mu, h) \leqslant 0$ whenever $0 \leqslant h \leqslant \mu \leqslant 0.6$.

Next, from our description of $\left\{c_{j}(\mu)\right\}$, it is clear that the function

$$
F(\mu):=f(\mu, \tanh \mu)(\cosh \mu)^{12} e^{16 \mu}
$$

is a polynomial of degree at most 16 in $e^{2 \mu}$. Hence $F\left(\frac{1}{2}(\log (x+1))\right)$ is a polynomial of degree at most 16 in $x$. It turns out that $F\left(\frac{1}{2}(\log (x+1))\right)$ is divisible by $x^{4}$, and one verifies that the quotient polynomial is positive for all $0 \leqslant x \leqslant 2.25$ (cf. [7]). Hence $F(\mu) \geqslant 0$ whenever $0 \leqslant \mu \leqslant$ $\frac{1}{2} \log (3.25)=0.5893 \ldots$ It follows that $f(\mu, \tanh \mu) \geqslant 0$ for all $\mu \in[0,0.58]$, and combining this with the fact that $(\partial / \partial h) f(\mu, h) \leqslant 0$ whenever $0 \leqslant h \leqslant \mu \leqslant 0.6$, we conclude that $f(\mu, h) \geqslant 0$, that is (A.16) holds, whenever $0 \leqslant \mu \leqslant 0.58$ and $0 \leqslant h \leqslant \tanh \mu$. Using $\tanh (0.58)<0.55$ it also follows that (A.13) holds for all such $(\mu, h)$ with $\mu>0$.

Finally, using interval arithmetic (first dividing through by $e^{4 \mu}$ ) we also prove that $(\partial / \partial h) f(\mu, h)<0$ for all $\mu \geqslant 0.58,0 \leqslant h \leqslant 0.5$, and also that $f(\mu, 0.5)>0$ for all $\mu \geqslant 0.58$ (cf. [7]). Combining these two facts it follows that $f(\mu, h)>0$ whenever $\mu \geqslant 0.58$ and $0 \leqslant$ $h \leqslant 0.5$. Using $\tanh (0.58)>0.5$ it follows that also (A.13) holds for all such $(\mu, h)$.

Case II, Step 2: Proof of (A.13) when $\mu \geqslant 1.5$ and $0.5 \leqslant h \leqslant \tanh \mu$.

Sublemma 6. For any fixed $0 \leqslant h \leqslant \mu$, the function

$$
x \mapsto-(\cosh (\mu-h)) x+(\cosh \mu) \arcsin x
$$

is increasing for $x \in[0,1]$.

Proof. Immediate by differentiation or otherwise.

Combining this sublemma with the fact that $\beta(h) \leqslant B(\mu, h)^{\frac{1}{2}} \leqslant 1$ where $\beta(h):=(1-(1-$ $\left.h)^{2} e^{2 h}\right)^{\frac{1}{2}}$ (cf. Sublemma 5), we see that (A.13) certainly holds at every point $(\mu, h)$ with $0 \leqslant h \leqslant \tanh \mu$ where the following function is non-negative:

$$
f(\mu, h):=-(\cosh (\mu-h)) \beta(h)+(\cosh \mu) \arcsin (\beta(h))-h^{2} \sinh \mu .
$$

Using interval arithmetic we prove that $f(1.5, h)>0$ and $(\partial / \partial \mu) f(1.5, h)>0$ for all $h \in$ $[0.5,1]$, cf. [7]. However we also note that $\partial^{2} f / \partial \mu^{2} \equiv f$. Hence for every fixed $h \in[0.5,1]$, it follows that $f(\mu, h)>0$ holds for all $\mu \geqslant 1.5$. Hence (A.13) indeed holds for all $\mu \geqslant 1.5$ and all $0.5 \leqslant h \leqslant \tanh \mu$.

Case II, Step 3: Proof of (A.13) when $0.58 \leqslant \mu \leqslant 1.5$ and $0.5 \leqslant h \leqslant \tanh \mu$. This is verified in [7] using brute force interval arithmetic; in fact we prove that (A.13) holds with strict inequality for all these $\mu, h$. This verification takes a few seconds on a $2.2 \mathrm{Ghz}$ PC. In order to calculate the difference of the two sides in (A.13) reasonably efficiently in interval arithmetic we make strong use of the monotonicity properties recorded in Sublemmas 5 and 6.

Note that steps $1-3$ together prove that (A.13) holds for all $\mu>0, h \in[0, \tanh \mu]$. This completes the treatment of case II, and hence also completes the proof of (19). 
Proof of Lemma 4, the inequality (20). As in the proof of (19) (case I) we see that our task is to prove

$$
(\cosh (\mu+h)) A^{\frac{1}{2}}-(\cosh \mu) \arcsin \left(A^{\frac{1}{2}}\right) \geqslant \frac{4 \sqrt{3}}{27} h^{3} \cosh \mu,
$$

for all $\mu, h \geqslant 0$, where $A=A(\mu, h)$ is again given by (A.6). Using Sublemmata 2 and 3 we see that (A.17) would follow if we could prove

$$
(\cosh (\mu+h)) A(0, h)^{\frac{1}{2}}-(\cosh \mu) \arcsin \left(A(0, h)^{\frac{1}{2}}\right) \geqslant \frac{4 \sqrt{3}}{27} h^{3} \cosh \mu .
$$

But we have $\cosh (\mu+h)=\cosh \mu \cosh h+\sinh \mu \sinh h \geqslant \cosh \mu \cosh h$; hence it suffices to prove that the following one-variable inequality holds for all $h \geqslant 0$ :

$$
(\cosh h) A(0, h)^{\frac{1}{2}}-\arcsin \left(A(0, h)^{\frac{1}{2}}\right) \geqslant \frac{4 \sqrt{3}}{27} h^{3} .
$$

We handle $h$ large by a crude analysis: from the Taylor series for $\cosh h$ we know that $\cosh h \geqslant \frac{1}{24} h^{4}$ for all $h \geqslant 0$. Hence, using again Sublemma 2 and $A(0,10)^{\frac{1}{2}}=0.999 \ldots>\frac{99}{100}$, we see that for every $h \geqslant 10$ the left-hand side of (A.18) is

$$
\geqslant \frac{1}{24} h^{4} A(0,10)^{\frac{1}{2}}-\frac{\pi}{2}>\frac{h^{4}-50}{25} \geqslant \frac{9 h^{3}+h^{3}-100}{25}>\frac{9 h^{3}}{25}>\frac{4 \sqrt{3}}{27} h^{3} .
$$

Hence (A.18) holds when $h \geqslant 10$.

For $1 \leqslant h \leqslant 10$ we verify that (A.18) holds, with strict inequality, using interval arithmetic, cf. [7]. Finally for $0 \leqslant h \leqslant 1$ we verify (A.18) by making appropriate use of Taylor expansions; again cf. [7].

Proof of Lemma 5(a). From (6) it follows that for $u \geqslant u_{\pi}, u \neq \mu$, we have

$$
v^{\prime}(u)=\operatorname{sgn}(u-\mu) \frac{T \sinh u-(T u-S) \cosh u}{\sinh u \sqrt{\sinh ^{2} u-(T u-S)^{2}}} .
$$

We will prove that $v^{\prime}(u)$ is strictly increasing by proving that $v^{\prime \prime}(u)>0$ for all $u \geqslant u_{\pi}, u \neq \mu$. (We remark that also $v^{\prime \prime}(\mu)=\frac{2}{3} \operatorname{coth} \mu>0$.)

Case I: Assume $0<\mu<u$. Differentiating once more in (A.19) we obtain

$$
v^{\prime \prime}(u)=\frac{f(\mu, h)}{(\sinh (\mu+h))^{2}\left(\sinh ^{2}(\mu+h)-(h \cosh \mu+\sinh \mu)^{2}\right)^{\frac{3}{2}}},
$$

where $h:=u-\mu>0$, and where $f(\mu, h)$ is a certain polynomial in $e^{\mu}, e^{-\mu}, e^{h}, e^{-h}, h$. It now suffices to prove that $f(\mu, h)>0$ for all $h>0$.

However, it turns out that $f(\mu, 0),(\partial / \partial h) f(\mu, 0)$ and $\left(\partial^{2} / \partial h^{2}\right) f(\mu, 0)$ all vanish identically, while $\left(\partial^{k} / \partial h^{k}\right) f(\mu, 0)$ for $k=3,4,5,6,7$ have simple factorizations which immediately show that they are positive for all $\mu>0$ (cf. [7]). Furthermore computing $\left(\partial^{8} / \partial h^{8}\right) f(\mu, h)$ and inspecting the formula immediately shows that $\left(\partial^{8} / \partial h^{8}\right) f(\mu, h)>0$ for all $\mu, h>0$ (cf. [7]). It follows from these facts that $f(\mu, h)>0$ for all $\mu, h>0$, as desired.

Case II: Assume $u_{\pi} \leqslant u<\mu$. Then again from (A.19) we obtain

$$
v^{\prime \prime}(u)=\frac{f(\mu, h)}{(\sinh (\mu-h))^{2}\left(\sinh ^{2}(\mu-h)-(\sinh \mu-h \cosh \mu)^{2}\right)^{\frac{3}{2}}},
$$

where $h:=\mu-u>0$ and $f(\mu, h)$ is a polynomial in $e^{\mu}, e^{-\mu}, e^{h}, e^{-h}, h$ (not the same as in case I), and it now suffices to prove that $f(\mu, h)>0$ for all $\mu>0$ and all $h \in(0, \tanh \mu]$. We remark that this case is rather delicate; for instance the inequality fails for all small $\mu$ if we increase $h$ by $O\left(\mu^{3}\right)$ from $h=\tanh \mu$ to $h=\mu$ : we have $f(\mu, \mu)<0$ for all small $\mu>0$ ! 
We start by proving that

$$
\frac{\partial}{\partial \mu}\left(e^{-5 \mu} f(\mu, h)\right) \geqslant 0 \quad \text { for all } \mu>0,0 \leqslant h \leqslant \min (1, \mu) .
$$

For this we use the Taylor expansion of $g(\mu, h):=e^{2 \mu}(\partial / \partial \mu)\left(e^{-5 \mu} f(\mu, h)\right)$ with respect to $h$, with Lagrange's error term:

$$
g(\mu, h)=\sum_{n=0}^{N-1} c_{n}(\mu) h^{n}+F_{N}(\mu, \xi) h^{N}
$$

where

$$
\begin{aligned}
& c_{n}(\mu)=\left.\frac{1}{n !} e^{2 \mu} \frac{\partial^{n+1}}{\partial h^{n} \partial \mu}\left(e^{-5 \mu} f(\mu, h)\right)\right|_{h=0}, \\
& F_{N}(\mu, h)=\frac{1}{N !} e^{2 \mu} \frac{\partial^{N+1}}{\partial h^{N} \partial \mu}\left(e^{-5 \mu} f(\mu, h)\right),
\end{aligned}
$$

and $\xi=\xi(\mu, h) \in[0, h]$. It turns out that $c_{3}(\mu), c_{4}(\mu), c_{5}(\mu), \ldots$ are polynomials of degree at most 4 in $e^{-2 \mu}$, and $c_{0}(\mu) \equiv c_{1}(\mu) \equiv c_{2}(\mu) \equiv 0$ (cf. [7]); thus $g(\mu, h)=0$ at $h=0$ while for $h>0$ we have

$$
h^{-3} g(\mu, h)=\sum_{n=3}^{N-1} c_{n}(\mu) h^{n-3}+F_{N}(\mu, \xi) h^{N-3} .
$$

Using interval arithmetic and splitting into sufficiently small $\mu, h$-boxes we prove that the right-hand side of (A.22) (with $N=12$ ) is positive for all $(\mu, h) \in[0.9, \infty) \times\left[0, \frac{1}{2}\right]$, cf. [7]. Similarly using the Taylor expansion around $h=\frac{1}{2}$ we also prove that $(\partial / \partial \mu)\left(e^{-5 \mu} f(\mu, h)\right)>0$ for all $(\mu, h) \in[0.9, \infty) \times\left[\frac{1}{2}, 1\right]$, cf. [7].

Hence to prove (A.20) it now remains to deal with the case $\mu<0.9$. The case of $(\mu, h)$ near $(0,0)$ is somewhat delicate, since $c_{3}(0)=c_{4}(0)=c_{5}(0)=c_{6}(0)=c_{7}(0)=0$ and $c_{8}(0)<0$ in (A.22); we have also noted experimentally that $(\partial / \partial \mu)\left(e^{-5 \mu} f\left(\mu, \frac{3}{2} \mu\right)\right)<0$ for all small $\mu>0$ ! To deal with this situation we substitute $\mu=-\frac{1}{2} \log (1-x)$ (namely $x=1-e^{-2 \mu}$ ) and $h=t x$ in (A.22). Then $c_{3}(\mu), c_{4}(\mu), \ldots$ are polynomials in $x$ of degree at most 4 , and it turns out that for $N=12$ we have

$$
\sum_{n=0}^{N-1} c_{n}(\mu) h^{n}=\sum_{n=0}^{N-1} c_{n}\left(-\frac{1}{2} \log (1-x)\right) \cdot(t x)^{n}=\sum_{j=6}^{15} P_{j}(t) x^{j},
$$

where each $P_{j}(t)$ is a polynomial in $t$ (with rational coefficients) which is divisible by $t^{3}$ and in particular $P_{6}(t)=\frac{1}{3} t^{3}\left(1-\frac{9}{4} t+t^{2}\right)$. It is crucial for our approach to work that $1-\frac{9}{4} t+t^{2}$ is bounded from below by a positive constant uniformly over $0 \leqslant t \leqslant \frac{1}{2}$.

We obtain

$$
\frac{g\left(-\frac{1}{2} \log (1-x), t x\right)}{t^{3} x^{6}}=\sum_{j=6}^{15}\left(t^{-3} P_{j}(t)\right) x^{j-6}+F_{12}\left(-\frac{1}{2} \log (1-x), \xi\right) t^{9} x^{6},
$$

where $\xi \in[0, t x]$, and where each $t^{-3} P_{j}(t)$ is a polynomial in $t$.

Using interval arithmetic and splitting the $t, x$-region into sufficiently small boxes we prove that the right-hand side of (A.24) is positive for all

$$
\begin{aligned}
(x, t) & \in([0,0.3] \times[0,0.6]) \cup([0.3,0.5] \times[0,0.7]) \cup([0.5,0.7] \times[0,0.86]) \\
& \cup([0.7,0.8] \times[0,1.01]) \cup([0.8,0.85] \times[0,1.12]) ;
\end{aligned}
$$

and one checks that this union in particular contains all $(x, t)$ with $0 \leqslant x \leqslant 0.85$ and $0 \leqslant$ $t \leqslant-\frac{1}{2} x^{-1} \log (1-x)$ (cf. [7]). Hence it follows that $g(\mu, h)>0$ holds for all $(\mu, h)$ with $0<\mu \leqslant-\frac{1}{2} \log (1-0.85)=0.948 \ldots$ and $0<h \leqslant \mu$, and the proof of (A.20) is complete. 
Next, we prove in [7], using Taylor expansion and interval arithmetic, that

$$
f\left(h+\frac{1}{3} h^{3}, h\right)>0, \quad \forall h \in(0,1] .
$$

Combining (A.25) and (A.20) we conclude that

$$
f(\mu, h)>0, \quad \forall 0<h \leqslant 1, \mu \geqslant h+\frac{1}{3} h^{3} .
$$

However $\operatorname{artanh} h=\sum_{k=0}^{\infty}(2 k+1)^{-1} h^{2 k+1}>h+\frac{1}{3} h^{3}$ for all $h \in(0,1)$, and hence it follows that $f(\mu, h)>0$ holds whenever $0<h<1$ and $\mu \geqslant \operatorname{artanh} h$. This completes the proof of Lemma $5(\mathrm{a})$.

Proof of Lemma 5(b). In view of Lemma 5(a) and the fact that $v^{\prime}(\mu)=-1$ (cf. $\S 2.1 .2$ ), Lemma $5(\mathrm{~b})$ will be proved if we can only show that $v^{\prime}\left(\frac{1}{2} \mu\right)>-2.9$, or equivalently (cf. (A.19)),

$$
\begin{aligned}
& \cosh \mu \sinh \left(\frac{1}{2} \mu\right)-\left(\sinh \mu-\frac{1}{2} \mu \cosh \mu\right) \cosh \left(\frac{1}{2} \mu\right) \\
& \quad<\frac{29}{10} \sinh \left(\frac{1}{2} \mu\right) \sqrt{\sinh ^{2}\left(\frac{1}{2} \mu\right)-\left(\sinh \mu-\frac{1}{2} \mu \cosh \mu\right)^{2}}
\end{aligned}
$$

for all $0<\mu \leqslant 1.8$. In [7] we prove this inequality by squaring, repeated differentiation and interval arithmetic.

Proof of Lemma 5(c). By Lemma 5a it suffices to prove that $v^{\prime}\left(u_{\pi}\right)>-3.3$ holds for all $\mu \geqslant 1.8$. Note that

$$
-v^{\prime}\left(u_{\pi}\right)=\frac{T}{\sinh u_{\pi}}=\frac{\cosh \mu}{\sinh (\mu-\tanh \mu)},
$$

and this function is decreasing as a function of $\mu$, since

$$
\frac{d}{d \mu}\left(\frac{\cosh \mu}{\sinh (\mu-\tanh \mu)}\right)=\frac{\sinh \mu \cosh (\mu-\tanh \mu)}{\sinh ^{2}(\mu-\tanh \mu)}(\tanh (\mu-\tanh \mu)-\tanh \mu)<0
$$

for all $\mu>0$. Hence the lemma follows from the fact that in the case when $\mu=1.8$, we have $v^{\prime}\left(u_{\pi}\right)=-3.23 \ldots>-3.3$.

Proof of Proposition 2. By differentiating under the integration sign in (1) and then moving to the path in (7) we obtain

$$
\begin{aligned}
\frac{\partial^{j_{1}+j_{2}}}{\partial r^{j_{1}} \partial x^{j_{2}}} K_{i r}(x)=\operatorname{Re}\{ & \int_{0}^{u_{c}}\left(i u-v\left(u_{c}\right)\right)^{j_{1}}\left(-\cosh \left(u+i v\left(u_{c}\right)\right)\right)^{j_{2}} e^{\phi\left(u+i v\left(u_{c}\right)\right)} d u \\
& \left.+\int_{u_{c}}^{\infty}(i u-v(u))^{j_{1}}(-\cosh (u+i v(u)))^{j_{2}} e^{\phi(u+i v(u))}\left(1+i v^{\prime}(u)\right) d u\right\} .
\end{aligned}
$$

We have $\cos v\left(u_{c}\right) \leqslant 0$ since $u_{c} \in\left[u_{\pi}, \mu\right]$, and from this it follows that $\operatorname{Re} \phi\left(u+i v\left(u_{c}\right)\right) \leqslant \eta\left(u_{c}\right)$ for all $u \in\left[0, u_{c}\right]$. Also note that $|\cosh (u+i v)|$ is an increasing function of $u \geqslant 0$ for any fixed $v \in \mathbb{R}$. Hence we obtain

$$
\begin{aligned}
\left|\frac{\partial^{j_{1}+j_{2}}}{\partial r^{j_{1}} \partial x^{j_{2}}} K_{i r}(x)\right| \leqslant & u_{c}\left|u_{c}+i v\left(u_{c}\right)\right|^{j_{1}}\left|\cosh \left(u_{c}+i v\left(u_{c}\right)\right)\right|^{j_{2}} e^{\eta\left(u_{c}\right)} \\
& +\int_{u_{c}}^{\infty}\left|1+i v^{\prime}(u)\right||u+i v(u)|^{j_{1}}|\cosh (u+i v(u))|^{j_{2}} e^{\eta(u)} d u
\end{aligned}
$$

We will now prove (24). Thus we assume $\varepsilon \leqslant x<r$. We will take $u_{c} \geqslant \frac{1}{2} \mu$ if $\mu<1.8$ and $u_{c}=u_{\pi}$ otherwise; hence by Lemma 5 we always have $\left|1+i v^{\prime}(u)\right| \ll 1$ for all $u \geqslant u_{c}$.

Let us first assume $\mu \geqslant 1.8$. Then $u_{c}:=u_{\pi}$; thus $\left(\mu-u_{c}\right)^{2} \geqslant(\tanh 1.8)^{2}>\frac{1}{2}$. Using also $|\cosh (u+i v)| \leqslant e^{|u|}$ and the first bound in Lemma 4, we obtain that (A.26) is

$$
\ll \mu^{j_{1}+1} e^{j_{2} \mu} e^{-(\pi / 2) r-\frac{1}{2} \sqrt{r^{2}-x^{2}}}+e^{-(\pi / 2) r} \int_{u_{c}}^{\infty} u^{j_{1}} e^{j_{2} u-\sqrt{r^{2}-x^{2}}(u-\mu)^{2}} d u .
$$


Recall here that we allow the implied constant to depend on $\varepsilon, j_{1}, j_{2}$ only. Replacing $u$ by $u+\mu+\frac{1}{2} j_{2}\left(r^{2}-x^{2}\right)^{-\frac{1}{2}}$ in the integral, and using $\mu \leqslant \log (2 r / x) \leqslant \log \left(2 \varepsilon^{-1} r\right)$ and $r^{2}-x^{2} \asymp$ $r^{2} \geqslant \varepsilon^{2}$ (which holds since $\mu \geqslant 1.8$ ), we obtain

$$
\begin{aligned}
& \ll e^{-(\pi / 2) r}\left\{e^{-\frac{1}{4} \sqrt{r^{2}-x^{2}}}+e^{j_{2} \mu} \int_{-\infty}^{\infty}\left(\mu^{j_{1}}+|u|^{j_{1}}\right) e^{-\sqrt{r^{2}-x^{2}} u^{2}} d u\right\} \\
& \ll e^{-(\pi / 2) r}\left(\frac{r}{x}\right)^{j_{2}}\left\{\left(r^{2}-x^{2}\right)^{-\frac{1}{4}}\left(\log \frac{2 r}{x}\right)^{j_{1}}+\left(r^{2}-x^{2}\right)^{-\left(j_{1}+1\right) / 4}\right\},
\end{aligned}
$$

and hence (24) holds in this case.

We now turn to the case $\mu<1.8$. Let us first take $u_{c}:=\frac{1}{2} \mu$. Recall $t_{0}^{+}=\mu+i \pi / 2$; thus $\cosh t_{0}^{+}=i \sinh \mu$, and for $\left|t-t_{0}^{+}\right|$bounded we have $\left|\cosh t-\cosh t_{0}^{+}\right| \ll\left|t-t_{0}^{+}\right|$(since $\mu<1.8$ ). Hence for all $u \in\left[u_{c}, 2\right]$ we have $|\cosh (u+i v(u))| \ll u$. Using again the first bound in Lemma 4 , we now get that (A.26) is:

$$
\begin{aligned}
& \ll \mu^{1+j_{2}} e^{-(\pi / 2) r-\frac{1}{4} \sqrt{r^{2}-x^{2}} \mu^{2}}+e^{-\frac{\pi}{2} r} \int_{\frac{1}{2} \mu}^{2} u^{j_{2}} e^{-\sqrt{r^{2}-x^{2}}(u-\mu)^{2}} d u \\
& +e^{-(\pi / 2) r} \int_{2}^{\infty} u^{j_{1}} e^{j_{2} u-\sqrt{r^{2}-x^{2}}(u-\mu)^{2}} d u \\
& \ll e^{-(\pi / 2) r}\left\{\mu^{1+j_{2}} e^{-\frac{1}{4} \sqrt{r^{2}-x^{2}} \mu^{2}}+\int_{-\infty}^{\infty}\left(\mu^{j_{2}}+|u|^{j_{2}}\right) e^{-\sqrt{r^{2}-x^{2}} u^{2}} d u\right. \\
& \left.\quad+\int_{2-\mu}^{\infty} u^{j_{1}} e^{j_{2} u-\sqrt{r^{2}-x^{2}} u^{2}} d u\right\} .
\end{aligned}
$$

Here

$$
\int_{-\infty}^{\infty}\left(\mu^{j_{2}}+|u|^{j_{2}}\right) e^{-\sqrt{r^{2}-x^{2}} u^{2}} d u \ll \frac{\mu^{j_{2}}}{\sqrt[4]{r^{2}-x^{2}}}+\left(r^{2}-x^{2}\right)^{-\left(j_{2}+1\right) / 4}
$$

Note that

$$
\mu \asymp \sqrt{\frac{r-x}{x}} \asymp \frac{\sqrt{r^{2}-x^{2}}}{x}, \quad \text { since } \mu<1.8 .
$$

Let us now also assume $x \leqslant r-r^{\frac{1}{3}}$. Then in the right-hand side of (A.28), the first term dominates the second. Using the fact that $a e^{-a^{2}}$ is uniformly bounded for all $a>0$ it follows that $\mu^{1+j_{2}} e^{-\frac{1}{4} \sqrt{r^{2}-x^{2}} \mu^{2}}$ is dominated by the right-hand side of (A.28); and since $r^{2}-x^{2} \gg r^{\frac{4}{3}} \geqslant \varepsilon^{\frac{4}{3}}$ it follows that the last integral in (A.27) is also dominated by the same expression. Hence we conclude that (24) holds also in the present case.

It now remains to treat the case when $\mu<1.8$ and $r-r^{\frac{1}{3}}<x<r$. By repeating the argument which led to (A.27) but taking $u_{c}=\mu$ and using the second bound in Lemma 4 instead of the first, we obtain that (A.26) is, writing $c=\frac{4 \sqrt{3}}{27}$,

$$
\ll e^{-(\pi / 2) r}\left\{\mu^{1+j_{2}}+\int_{\mu}^{2} u^{j_{2}} e^{-c r(u-\mu)^{3}} d u+\int_{2}^{\infty} u^{j_{1}} e^{j_{2} u-c r(u-\mu)^{3}} d u\right\} .
$$

Here the first integral is

$$
\ll \int_{0}^{\infty}\left(\mu^{j_{2}}+u^{j_{2}}\right) e^{-c r u^{3}} d u \ll \mu^{j_{2}} r^{-\frac{1}{3}}+r^{-\frac{1}{3}\left(j_{2}+1\right)},
$$

and the last integral is $O\left(-\frac{1}{3}\left(j_{2}+1\right)\right)$, since $r \geqslant \varepsilon$. Note also that $\mu \asymp \sqrt{(r-x) / x} \ll r^{-\frac{1}{3}}$ because $\mu<1.8$ and $r-r^{\frac{1}{3}}<x<r$. Hence we conclude that (24) holds also in this last case.

We now turn to the proof of (21)-(23). By Lemma $5\left(\right.$ a) we have $-1 \leqslant v^{\prime}(u)<0$ for all $u \geqslant \mu$. Assume that we have chosen $u_{c} \in\left[u_{\pi}, \mu\right]$ in such a way that $-C<v^{\prime}(u) \leqslant-1$ for all $u \in\left[u_{c}, \mu\right]$. 
Then for any $j \geqslant 0$ we have, by (A.26),

$$
\begin{aligned}
\left|\frac{\partial^{j}}{\partial r^{j}} K_{i r}(x)\right| \leqslant & u_{c}\left|u_{c}+i v\left(u_{c}\right)\right|^{j} e^{\eta\left(u_{c}\right)} \\
& +|1+i C||\mu+i \pi|^{j} \int_{u_{c}}^{\mu} e^{\eta(u)} d u+\sqrt{2} \int_{\mu}^{\infty}\left|u+i \frac{\pi}{2}\right|^{j} e^{\eta(u)} d u .
\end{aligned}
$$

For $j=1$ we use here $|\mu+i \pi|<\mu+\pi$ and $|u+i \pi / 2|<u+\pi / 2$. Then, using the first bound in Lemma 4 and extending the integral $\int_{u_{c}}^{\mu}$ to $\int_{-\infty}^{\mu}$, we find that the last line in (A.29) is bounded above by $e^{-(\pi / 2) r}$ times

$$
\begin{cases}\frac{\frac{1}{2}|1+i C| \sqrt{\pi}+\sqrt{\pi / 2}}{\sqrt[4]{r^{2}-x^{2}}} & (j=0), \\ \frac{\frac{1}{2}|1+i C|(\mu+\pi) \sqrt{\pi}+\sqrt{\pi / 2} \mu+(\pi / 2)^{\frac{3}{2}}}{\sqrt[4]{r^{2}-x^{2}}}+\frac{1}{\sqrt{2} \sqrt{r^{2}-x^{2}}} & (j=1), \\ \frac{\frac{1}{2}|1+i C|\left(\mu^{2}+\pi^{2}\right) \sqrt{\pi}+\sqrt{\pi / 2} \mu^{2}+(\pi / 2)^{\frac{5}{2}}}{\sqrt[4]{r^{2}-x^{2}}}+\frac{\sqrt{2} \mu}{\sqrt{r^{2}-x^{2}}}+\frac{2^{-\frac{3}{2}} \sqrt{\pi}}{\left(r^{2}-x^{2}\right)^{\frac{3}{2}}} & (j=2) .\end{cases}
$$

Let us first assume $\mu \geqslant 1.8$. In this case we take $u_{c}=u_{\pi}$. Now (A.29) holds with $C=3.3$, by Lemma $5(\mathrm{c})$. We also have $r / x=\cosh \mu>3$; thus $\sqrt{r^{2}-x^{2}}>\sqrt{8 / 9} r$; also $\left(\mu-u_{c}\right)^{2}=$ $\tanh ^{2} \mu>0.89$ so that $\eta\left(u_{c}\right)<-(\pi / 2) r-0.89 \sqrt{r^{2}-x^{2}}$, and $u_{c}<\mu<\log (2 r / x) \leqslant \log (2 r)$. Hence we see that the first term in the right-hand side of (A.29) is bounded above by (cf. [7]):

$$
\begin{aligned}
& \log (2 r) e^{-(\pi / 2) r-0.89 \sqrt{8 / 9} r}<e^{-(\pi / 2) r} \frac{0.4}{\sqrt{r}}<e^{-(\pi / 2) r} \frac{0.4}{\sqrt[4]{r^{2}-x^{2}}} \quad(\text { if } j=0) \\
& \log (2 r)(\pi+\log (2 r)) e^{-(\pi / 2) r-0.89 \sqrt{8 / 9} r}<e^{-(\pi / 2) r} \frac{1.7}{\sqrt[4]{r^{2}-x^{2}}} \quad(\text { if } j=1) \\
& \log (2 r)\left(\pi^{2}+(\log 2 r)^{2}\right) e^{-(\pi / 2) r-0.89 \sqrt{8 / 9} r}<e^{-(\pi / 2) r} \frac{4.4}{\sqrt[4]{r^{2}-x^{2}}} \quad(\text { if } j=2) .
\end{aligned}
$$

Adding up these, and using (for $j=1,2) \sqrt[4]{r^{2}-x^{2}}>\sqrt[4]{\left(3^{2}-1\right) x^{2}} \geqslant \sqrt[4]{8}$ and (for $j=2$ ) $\log (r / x) \leqslant \frac{1}{2}+\frac{1}{2}(\log (r / x))^{2}$, we obtain:

$$
\begin{aligned}
\left|K_{i r}(x)\right| & <e^{-(\pi / 2) r} \frac{5}{\sqrt[4]{r^{2}-x^{2}}} ; \\
\left|\frac{\partial}{\partial r} K_{i r}(x)\right| & <e^{-(\pi / 2) r} \frac{17+5 \log (r / x)}{\sqrt[4]{r^{2}-x^{2}}} ; \\
\left|\frac{\partial^{2}}{\partial r^{2}} K_{i r}(x)\right| & <e^{-(\pi / 2) r} \frac{44+8 \log (r / x)^{2}}{\sqrt[4]{r^{2}-x^{2}}} .
\end{aligned}
$$

Next assume $\mu<1.8$. We then take $u_{c}=\frac{1}{2} \mu$, and by Lemma 5 (b), (A.29) holds with $C=2.9$. Also, the first term in the right-hand side of (A.29) is now bounded above by, if $j=0$ :

$$
\frac{\mu}{2} e^{-(\pi / 2) r-\frac{1}{4} \mu^{2} \sqrt{r^{2}-x^{2}}} \leqslant e^{-(\pi / 2) r} \frac{(2 e)^{-\frac{1}{2}}}{\sqrt[4]{r^{2}-x^{2}}}
$$

where we used the fact that $t e^{-t^{2}} \leqslant(2 e)^{-\frac{1}{2}}$ for all $t>0$. In the case $j=1(j=2)$ we get the same bound times a factor $\mu / 2+\pi<0.9+\pi$, (times a factor $\left.(\mu / 2)^{2}+\pi^{2}<0.81+\pi^{2}\right)$. Adding up our bounds for $j=0$ we again obtain that the first line of (A.30) holds, that is this bound on $\left|K_{i r}(x)\right|$ holds for all $1 \leqslant x<r$. For $j=1,2$ we make the further assumption that $x \leqslant r-\frac{1}{2} r^{\frac{1}{3}}$; then we have $r^{2}-x^{2} \geqslant r^{2}-\left(r-\frac{1}{2} r^{\frac{1}{3}}\right)^{2}=r^{\frac{4}{3}}\left(1-\frac{1}{4} r^{-\frac{2}{3}}\right)>\frac{3}{4}$; using this and adding up the bounds we also find that the second and third line of (A.30) hold. 
It now remains to treat the case $r-\frac{1}{2} r^{\frac{1}{3}} \leqslant x<r$. Then

$$
\mu=\operatorname{arcosh}(T)<\sqrt{2(T-1)}=\sqrt{\frac{2(r-x)}{x}} \leqslant \sqrt{\frac{2 \cdot \frac{1}{2} r^{\frac{1}{3}}}{\frac{1}{2} r}}=\sqrt{2} r^{-\frac{1}{3}} .
$$

(In particular $\mu<1.8$ holds automatically.) In this case we take $u_{c}=\mu$ in (A.29). Now for $j=0$ the first term in the right-hand side of (A.29) is less than $\sqrt{2} r^{-\frac{1}{3}} e^{-(\pi / 2) r}$. For $j=1 \quad(j=2)$ we get the same bound times a factor $\mu+\pi / 2<\sqrt{2}+\pi / 2$ (times a factor $\left.\mu^{2}+(\pi / 2)^{2}<2+(\pi / 2)^{2}\right)$. Also the middle term in (A.29) vanishes, and in the last term we use the second bound in Lemma 4 , and for $j=1$ we also use $|u+i \pi / 2| \leqslant u+\pi / 2$; after this the integral can be evaluated in exact terms. Adding up the contributions we obtain the bounds stated in Proposition 2.

Our next task is to prove Lemma 7. We first prove some auxiliary results.

Sublemma 7. The generating function of the Jacobi polynomials $P_{n}^{(s,-s)}(x)$ can be expressed in terms of the Legendre polynomials as follows:

$$
\sum_{n=0}^{\infty} P_{n}^{(s,-s)}(x) t^{n}=\sum_{n=0}^{\infty} P_{n}(x) t^{n} \exp \left(s \int_{0}^{t} \sum_{m=0}^{\infty} P_{m}(x) u^{m} d u\right) .
$$

Proof. The generating function of the Jacobi polynomials reads [36, equation (4.4.5)]

$$
\sum_{n=0}^{\infty} P_{n}^{(s,-s)}(x) t^{n}=\left(1-2 x t+t^{2}\right)^{-\frac{1}{2}}\left(\frac{1+t+\left(1-2 x t+t^{2}\right)^{\frac{1}{2}}}{1-t+\left(1-2 x t+t^{2}\right)^{\frac{1}{2}}}\right)^{s} .
$$

Identifying

$$
\frac{\partial}{\partial t} \log \left(\frac{1+t+\left(1-2 x t+t^{2}\right)^{\frac{1}{2}}}{1-t+\left(1-2 x t+t^{2}\right)^{\frac{1}{2}}}\right)=\left(1-2 x t+t^{2}\right)^{-\frac{1}{2}}
$$

with the generating function of the Legendre polynomials

$$
\sum_{n=0}^{\infty} P_{n}(x) t^{n}=\left(1-2 x t+t^{2}\right)^{-\frac{1}{2}}
$$

gives the desired result.

Sublemma 8. (a) For each $n, j \in \mathbb{Z}_{\geqslant 0},\left.\left(\partial^{j} / \partial s^{j}\right) P_{n}^{(s,-s)}(x)\right|_{s=0}$ is a non-negative linear combination of products of Legendre polynomials.

(b) Any product of Legendre polynomials is a non-negative linear combination of Legendre polynomials.

Proof. Using Sublemma 7 we obtain

$$
\left.\frac{\partial^{j}}{\partial s^{j}} \sum_{n=0}^{\infty} P_{n}^{(s,-s)}(x) t^{n}\right|_{s=0}=\sum_{n=0}^{\infty} P_{n}(x) t^{n}\left(\sum_{m=0}^{\infty} P_{m}(x) \frac{t^{m+1}}{m+1}\right)^{j} .
$$

Equating coefficients with respect to $t^{n}$ proves part (a) of Sublemma 8.

Using $\left\langle P_{n} P_{m}, P_{k}\right\rangle \geqslant 0$ iteratively yields part (b) of the sublemma.

Proof of Lemma 7. According to Sublemma 8, $\left.\left(\partial^{j} / \partial s^{j}\right) P_{n}^{(s,-s)}(x)\right|_{s=0}$ is a non-negative linear combination of Legendre polynomials. Applying the bound $\left|P_{k}(x)\right| \leqslant 1=P_{k}(1)$ for $x \in[-1,1]$ results in

$$
\left|\frac{\partial^{j}}{\partial s^{j}} P_{n}^{(s,-s)}(x)\right|_{s=0}\left|\leqslant \frac{\partial^{j}}{\partial s^{j}} P_{n}^{(s,-s)}(1)\right|_{s=0} .
$$

This establishes the lemma for $r=0$. 
Writing

$$
\begin{aligned}
& f_{n, j}(x, s):=n ! \frac{\partial^{j}}{\partial s^{j}} P_{n}^{(-s, s)}(x) \quad \text { for } n \in \mathbb{Z}_{\geqslant 0}, j \in \mathbb{Z}_{\geqslant 0}, \\
& f_{n, j}^{\prime}(x, s)=\frac{\partial}{\partial x} f_{n, j}(x, s), \quad f_{n, j}^{\prime \prime}(x, s)=\frac{\partial^{2}}{\partial x^{2}} f_{n, j}(x, s),
\end{aligned}
$$

and using the convention $f_{n,-1}(x, s)=0$, we have

$$
\left(1-x^{2}\right) f_{n, j}^{\prime \prime}+2 j f_{n, j-1}^{\prime}+(2 s-2 x) f_{n, j}^{\prime}+n(n+1) f_{n, j}=0, \quad \forall n \geqslant 0, j \geqslant 0,
$$

cf. [36, equation (4.2.1)].

If $n=0$ then $f_{n, j}(x, s)$ is a constant and (34) holds trivially.

Now assume that $n \geqslant 1$ and let

$$
n(n+1) g(x)=n(n+1)\left|f_{n, j}(x, s)\right|^{2}+\left(1-x^{2}\right)\left|f_{n, j}^{\prime}(x, s)\right|^{2} .
$$

Then we have $\left|f_{n, j}(x, s)\right|^{2} \leqslant g(x)$ for $x \in[-1,1]$, and $g(1)=g(-1)=\left|f_{n, j}(1, s)\right|^{2}$. Thus, it suffices to show that $g(x)$ attains its maximum on $[-1,1]$ at the endpoints.

Now on account of (A.32), cf. [36, p. 160],

$$
\begin{aligned}
& n(n+1) g^{\prime}(x)=\overline{f_{n, j}^{\prime}}\left\{n(n+1) f_{n, j}-x f_{n, j}^{\prime}+\left(1-x^{2}\right) f_{n, j}^{\prime \prime}\right\} \\
& +f_{n, j}^{\prime}\left\{n(n+1) \overline{f_{n, j}}-x \overline{f_{n, j}^{\prime}}+\left(1-x^{2}\right) \overline{f_{n, j}^{\prime \prime}}\right\} \\
& =\overline{f_{n, j}^{\prime}}\left\{-2 j f_{n, j-1}^{\prime}+(x-2 s) f_{n, j}^{\prime}\right\}+f_{n, j}^{\prime} \overline{\left\{-2 j f_{n, j-1}^{\prime}+(x-2 s) f_{n, j}^{\prime}\right\}} \\
& = \begin{cases}2 x\left|f_{n, 0}^{\prime}\right|^{2} & \text { if } s \in i \mathbb{R} \text { and } j=0, \\
x((n+1) !)^{2}\left(n^{2}+n-1\right) / 2 & \text { if } s \in i \mathbb{R} \text { and } j=n-1, \\
0 & \text { if } s \in i \mathbb{R} \text { and } j \geqslant n,\end{cases}
\end{aligned}
$$

so that $g(x)$ is decreasing for $x<0$ and increasing for $x>0$, provided $s \in i \mathbb{R}$ and $j=0$ or $j \geqslant n-1$. This establishes the lemma for $j=0$ and also for $j \geqslant n-1$.

Let $S_{l}, l=0,1,2, \ldots$ be the Stirling polynomials, defined via the generating function

$$
\sum_{l=0}^{\infty} \frac{S_{l}(x)}{l !} t^{l}=\left(\frac{t}{1-e^{-t}}\right)^{1+x}
$$

Then for any integer $n \geqslant l$ we have

$$
S_{l}(n)\left(\begin{array}{l}
n \\
l
\end{array}\right)=\left[\begin{array}{c}
n+1 \\
n+1-l
\end{array}\right],
$$

where the brackets are unsigned Stirling numbers of the first kind, given by

$$
\sum_{l=0}^{n}\left[\begin{array}{c}
n+1 \\
n+1-l
\end{array}\right] s^{n-l}=\prod_{\ell=1}^{n}(\ell+s)
$$

Turning to the Jacobi polynomials, we have

$$
\begin{aligned}
P_{n}^{(s,-s)}(x) & =\sum_{k=0}^{n}\left(\frac{x-1}{2}\right)^{k}\left(\begin{array}{c}
n+k \\
k
\end{array}\right) \frac{1}{(n-k) !} \prod_{\ell=1}^{n-k}(\ell+k+s) \\
& =\sum_{k=0}^{n}\left(\frac{x-1}{2}\right)^{k}\left(\begin{array}{c}
n+k \\
k
\end{array}\right) \sum_{l=0}^{n-k} \frac{S_{l}(n-k)}{l !} \frac{(s+k)^{n-k-l}}{(n-k-l) !} .
\end{aligned}
$$

Taking the $j$ th derivative and writing $j=n-m \geqslant 0$, we obtain

$$
\left(\frac{\partial}{\partial s}\right)^{n-m} P_{n}^{(s,-s)}(x)=\sum_{k=0}^{m}\left(\frac{x-1}{2}\right)^{k}\left(\begin{array}{c}
n+k \\
k
\end{array}\right) \sum_{l=0}^{m-k} \frac{S_{l}(n-k)}{l !} \frac{(s+k)^{m-k-l}}{(m-k-l) !}
$$


which is a polynomial in $n$ and $s$ of total degree at most $m$. Therefore,

$$
\left\langle\left|\left(\frac{\partial}{\partial r}\right)^{n-m} P_{n}^{(-i r, i r)}\right|^{2}, P_{k}\right\rangle
$$

is an even polynomial in $r$ of degree at most $2 m$. With the aid of computer algebra [7] (here we used PARI/GP [31]), we have verified that the coefficients of this polynomial are all nonnegative for $m \leqslant 100, m \leqslant n \leqslant 3 m, k \leqslant 2 m$. Moreover, since the coefficients are themselves polynomials in $n$ of degree at most $2 m$, we may employ the method of successive differences to prove that they are non-negative for any $n \geqslant m, m=n-j \leqslant 100$. This establishes the lemma for $n \leqslant j+100$ and completes the proof.

Proof of Proposition 4. Let us consider the integral $\int_{-\infty}^{\infty} \cos (x \sinh t) g(t) e^{s t} d t$ for some test function $g$ of Schwartz class. Writing $g(t)=\int_{-\infty}^{\infty} \hat{g}(r) e^{i r t} d r$, this is

$$
\begin{aligned}
\int_{-\infty}^{\infty} \cos (x \sinh t) g(t) e^{s t} d t \\
=\int_{-\infty}^{\infty} \int_{-\infty}^{\infty} \cos (x \sinh t) \hat{g}(r) e^{(s+i r) t} d r d t \\
=\int_{-\infty}^{\infty} \hat{g}(r) \int_{-\infty}^{\infty} \cos (x \sinh t) e^{(s+i r) t} d t d r, \quad \text { cf. (32), } \\
=\int_{-\infty}^{\infty} \hat{g}(r) \int_{-\infty}^{\infty} \frac{\cos ^{(-n)}(x \sinh t)}{(x \cosh t)^{n}} f_{n}(\tanh t, s+i r) e^{(s+i r) t} d t d r, \quad \text { cf. (33), } \\
=\int_{-\infty}^{\infty} \frac{\cos ^{(-n)}(x \sinh t)}{(x \cosh t)^{n}} e^{s t} \int_{-\infty}^{\infty} \hat{g}(r) f_{n}(\tanh t, s+i r) e^{i r t} d r d t \\
=\int_{-\infty}^{\infty} \frac{\cos ^{(-n)}(x \sinh t)}{(x \cosh t)^{n}} e^{s t} \int_{-\infty}^{\infty} \hat{g}(r) \sum_{j=0}^{n} \frac{\partial^{j} f_{n}}{\partial s^{j}}(\tanh t, s) \frac{(i r)^{j}}{j !} e^{i r t} d r d t \\
=\int_{-\infty}^{\infty} \frac{\cos ^{(-n)}(x \sinh t)}{(x \cosh t)^{n}} e^{s t} \sum_{j=0}^{n} \frac{g^{(j)}(t)}{j !} \frac{\partial^{j} f_{n}}{\partial s^{j}}(\tanh t, s) d t .
\end{aligned}
$$

With $w(r)=e^{-\left(r-r_{0}\right)^{2} / 2 h^{2}}$ and $F(r)=\widetilde{K}_{i r}(x) w(r)$, we have

$$
\hat{F}(u)=\int_{-\infty}^{\infty} \cos (x \sinh t) \hat{w}(u-t) d t=\frac{h}{\sqrt{2 \pi}} e^{-i r_{0} u} \int_{-\infty}^{\infty} \cos (x \sinh t) g_{u}(t) e^{i r_{0} t} d t
$$

where $\hat{w}(t)=(h / \sqrt{2 \pi}) e^{-i r_{0} t} e^{-h^{2} t^{2} / 2}$ and $g_{u}(t)=e^{-h^{2}(u-t)^{2} / 2}$. Now, $g_{u}^{(j)}(t)=h^{j} H_{j}(h(u-$ t) $g_{u}(t)$, where $H_{j}$ is the $j$ th Hermite polynomial. Thus, by (A.33), we obtain

$$
\begin{aligned}
\hat{F}(u)= & \frac{h}{\sqrt{2 \pi}} \int_{-\infty}^{\infty} \frac{\cos ^{(-n)}(x \sinh t)}{(x \cosh t)^{n}} \sum_{j=0}^{n} \frac{h^{j}}{j !} H_{j}(h(u-t)) e^{-h^{2}(u-t)^{2} / 2} \\
& \times \frac{\partial^{j} f_{n}}{\partial s^{j}}\left(\tanh t, i r_{0}\right) e^{-i r_{0}(u-t)} d t \\
= & \frac{x^{-n}}{\sqrt{2 \pi}} \int_{-\infty}^{\infty} \frac{\cos ^{(-n)}(x \sinh (u-t / h))}{\cosh (u-t / h)^{n}} \sum_{j=0}^{n} \frac{h^{j}}{j !} H_{j}(t) e^{-t^{2} / 2} \\
& \times \frac{\partial^{j} f_{n}}{\partial s^{j}}\left(\tanh (u-t / h), i r_{0}\right) e^{-i r_{0} t / h} d t .
\end{aligned}
$$


By Cauchy-Schwarz and Conjecture 1, for any $a>0$ we have

$$
\begin{aligned}
x^{2 n}|\hat{F}(u)|^{2} \leqslant & \int_{-\infty}^{\infty} \sum_{j=0}^{n}\left(\frac{(a h)^{j}}{j !} H_{j}(t)\right)^{2} \frac{e^{-t^{2} / 2}}{\cosh (u-t / h)^{n}} \frac{d t}{\sqrt{2 \pi}} \\
& \times \int_{-\infty}^{\infty} \sum_{j=0}^{n} a^{-2 j}\left|\frac{\partial^{j} f_{n}}{\partial s^{j}}\left(1, i r_{0}\right)\right|^{2} \frac{e^{-t^{2} / 2}}{\cosh (u-t / h)^{n}} \frac{d t}{\sqrt{2 \pi}}
\end{aligned}
$$

Using the crude bound $\cosh (u-t / h)^{-1} \leqslant 2 e^{-u+t / h}$, we have

$$
\begin{aligned}
\int_{-\infty}^{\infty} H_{j}(t)^{2} \frac{e^{-t^{2} / 2}}{\cosh (u-t / h)^{n}} \frac{d t}{\sqrt{2 \pi}} & \leqslant 2^{n} e^{-n u} \int_{-\infty}^{\infty} H_{j}(t)^{2} e^{-t^{2} / 2+n t / h} \frac{d t}{\sqrt{2 \pi}} \\
& =2^{n} e^{-n u+n^{2} / 2 h^{2}} \int_{-\infty}^{\infty} H_{j}(t+n / h)^{2} e^{-t^{2} / 2} \frac{d t}{\sqrt{2 \pi}}
\end{aligned}
$$

Using the identity $H_{j}(x+y)=\sum_{k=0}^{j}\left(\begin{array}{l}j \\ k\end{array}\right) y^{j-k} H_{k}(x)$ and orthogonality of the Hermite polynomials, the last line equals

$$
\begin{aligned}
& 2^{n} e^{-n u+n^{2} / 2 h^{2}} \int_{-\infty}^{\infty}\left(\sum_{k=0}^{j}\left(\begin{array}{l}
j \\
k
\end{array}\right)\left(\frac{n}{h}\right)^{j-k} H_{k}(t)\right)^{2} e^{-t^{2} / 2} \frac{d t}{\sqrt{2 \pi}} \\
& =2^{n} e^{-n u+n^{2} / 2 h^{2}} \int_{-\infty}^{\infty} \sum_{k=0}^{j}\left(\begin{array}{l}
j \\
k
\end{array}\right)^{2}\left(\frac{n}{h}\right)^{2 j-2 k} H_{k}(t)^{2} e^{-t^{2} / 2} \frac{d t}{\sqrt{2 \pi}} \\
& =2^{n} e^{-n u+n^{2} / 2 h^{2}} \sum_{k=0}^{j}\left(\begin{array}{l}
j \\
k
\end{array}\right)^{2} k !\left(\frac{n}{h}\right)^{2 j-2 k} \\
& =2^{n} e^{-n u+n^{2} / 2 h^{2}} j ! L_{j}\left(-n^{2} / h^{2}\right),
\end{aligned}
$$

where $L_{j}$ is the $j$ th Laguerre polynomial. Taking $j=0$ gives the bound $2^{n} e^{-n u+n^{2} / 2 h^{2}}$ for $\int\left(e^{-t^{2} / 2} / \cosh (u-t / h)^{n}\right)(d t / \sqrt{2 \pi})$. On the other hand, from the identity $\sum_{j=0}^{\infty}\left(z^{j} / j !\right) L_{j}(-x)=$ $e^{z} I_{0}(2 \sqrt{x z})$, we obtain

$$
\begin{aligned}
& \int_{-\infty}^{\infty} \sum_{j=0}^{n}\left(\frac{(a h)^{j}}{j !} H_{j}(t)\right)^{2} \frac{e^{-t^{2} / 2}}{\cosh (u-t / h)^{n}} \frac{d t}{\sqrt{2 \pi}} \\
& \quad \leqslant 2^{n} e^{-n u+n^{2} / 2 h^{2}} \sum_{j=0}^{n} \frac{(a h)^{2 j}}{j !} L_{j}\left(-n^{2} / h^{2}\right) \leqslant 2^{n} e^{-n u+n^{2} / 2 h^{2}+a^{2} h^{2}} I_{0}(2 a n) \\
& \leqslant 2^{n} e^{-n u+n^{2} / 2 h^{2}+2 a n+a^{2} h^{2}} .
\end{aligned}
$$

Thus, we have

$$
|\hat{F}(u)| \leqslant 2^{n} x^{-n} e^{-n u+(n / h+a h)^{2} / 2} \sqrt{\sum_{j=0}^{n} a^{-2 j}\left|\frac{\partial^{j} f_{n}}{\partial s^{j}}\left(1, i r_{0}\right)\right|^{2}} .
$$

Next, it is not hard to see that

$$
\left|\frac{\partial^{j} f_{n}}{\partial s^{j}}\left(1, i r_{0}\right)\right| \leqslant \frac{n !}{(n-j) !} \prod_{\ell=j+1}^{n}\left|\ell+i r_{0}\right| \leqslant \frac{n !}{(n-j) !} R^{-j} \prod_{\ell=1}^{n}|\ell+i R|,
$$

for any $R \in \mathbb{R}_{>0}$ with $R \geqslant\left|r_{0}\right|$. Thus,

$$
\sum_{j=0}^{n} a^{-2 j}\left|\frac{\partial^{j} f_{n}}{\partial s^{j}}\left(1, i r_{0}\right)\right|^{2} \prod_{\ell=1}^{n}\left(\ell^{2}+R^{2}\right)^{-1} \leqslant \sum_{j=0}^{n}\left(\frac{n !}{(n-j) !}\right)^{2}(a R)^{-2 j}
$$


Now take $a=n / R$; then the last formula becomes

$$
\sum_{j=0}^{n}\left(\frac{n !}{(n-j) ! n^{j}}\right)^{2} \leqslant \sum_{j=0}^{n} \exp \left(-\frac{j(j-1)}{n}\right) \leqslant 2 \sqrt{n} .
$$

Also using the estimate $\prod_{\ell=1}^{n}\left(\ell^{2}+R^{2}\right) \leqslant R^{2 n} \exp \left(n(n+1)(2 n+1) /\left(6 R^{2}\right)\right)$, we finally have

$$
|\hat{F}(u)| \leqslant(4 n)^{1 / 4} \exp \left[\frac{n(n+1)(2 n+1)}{12 R^{2}}+\frac{1}{2} n^{2}\left(\frac{1}{h}+\frac{h}{R}\right)^{2}+n \log \frac{2 R}{x}-n u\right] .
$$

Proof of Proposition 5. Suppose that we have an inequality of the form $\left|\widetilde{K}_{i r}(x)\right| \leqslant C x^{-1 / 3}$ for an absolute constant $C$; we will return to this point below. Using this bound for $\widetilde{K}_{i r}(x)$ together with the estimate

$$
\left|\operatorname{sinc}^{(\ell)}(x)\right| \leqslant|x|^{-1}\left(1+x^{-2}\right)^{\ell / 2}, \quad \ell \in\{0,1\},
$$

the left-hand side of (36) is majorized by

$$
\begin{aligned}
& \frac{2 C}{\pi M x^{1 / 3}}\left(X \sqrt{\pi^{2}+M^{-2}}\right)^{\ell} \sum_{m=0}^{\infty} e^{-(m+M)^{2} /\left(2 h^{2} X^{2}\right)} \\
& <\frac{2 C}{\pi M x^{1 / 3}}\left(X \sqrt{\pi^{2}+M^{-2}}\right)^{\ell} e^{-M^{2} /\left(2 h^{2} X^{2}\right)} \sum_{m=0}^{\infty} e^{-2 M m /\left(2 h^{2} X^{2}\right)} \\
& =\frac{2 C}{\pi M x^{1 / 3}}\left(X \sqrt{\pi^{2}+M^{-2}}\right)^{\ell} \frac{e^{-M^{2} /\left(2 h^{2} X^{2}\right)}}{1-e^{-M /\left(h^{2} X^{2}\right)}} .
\end{aligned}
$$

Turning to the inequality for $\widetilde{K}_{i r}$, by symmetry we may assume that $r \geqslant 0$. Let us suppose first that $r \geqslant 1$. Then when $x \geqslant r$ we have from Proposition 1 that

$$
0<\widetilde{K}_{i r}(x) x^{1 / 3} \leqslant 2 \cosh \left(\frac{\pi r}{2}\right) e^{-(\pi / 2) r} \frac{\Gamma\left(\frac{1}{3}\right)}{2^{\frac{2}{3}} 3^{\frac{1}{6}}} e^{-r u(x / r)}\left(\frac{x}{r}\right)^{1 / 3}<\frac{3}{2} e^{-u(x / r)}\left(\frac{x}{r}\right)^{1 / 3},
$$

where $u(t)=\sqrt{t^{2}-1}-\arctan \sqrt{t^{2}-1}$ for $t \geqslant 1$. It is not hard to see that the function $\frac{3}{2} t^{1 / 3} e^{-u(t)}$ is maximum at $t=\sqrt{10 / 9}$, and its value there is comfortably less than 2 .

In the case $1 \leqslant x<r$ we apply Proposition 2 . Note that $x \leqslant r-\frac{1}{2} r^{\frac{1}{3}}$ implies $r^{2}-x^{2} \geqslant$ $r^{\frac{4}{3}}\left(1-\frac{1}{4} r^{-\frac{2}{3}}\right)>\frac{3}{4} r^{\frac{4}{3}} ;$ hence for any $1 \leqslant x<r$ we have

$$
\left|\widetilde{K}_{i r}(x)\right|<2 \cosh \left(\frac{\pi r}{2}\right) e^{-(\pi / 2) r} \cdot 5\left(\frac{4}{3}\right)^{1 / 4} r^{-1 / 3}<6 r^{-1 / 3}<6 x^{-1 / 3} .
$$

For $r \geqslant 1, x<1$ we use the identity $|\Gamma(1+i r)|=\sqrt{\pi r / \sinh (\pi r)}$ in the defining series (25) to derive the bound

$$
\left|\widetilde{K}_{i r}(x)\right| \leqslant \sqrt{\frac{2 \pi}{r \tanh (\pi r / 2)}} I_{0}(x)<4 x^{-1 / 3} .
$$

It only remains to handle the case of $r<1$. Applying (33) with $n=1$ (for which $f_{n}(\xi, s)=$ $\xi-s)$ we obtain

$$
\left|\widetilde{K}_{i r}(x)\right| \leqslant \frac{\sqrt{1+r^{2}}}{x} \int_{-\infty}^{\infty} \frac{d t}{\cosh t} \leqslant \pi \sqrt{2} x^{-1}<5 x^{-1 / 3}
$$

for $r<1$ and $x \geqslant 1$. For $x<1$ we have

$$
\begin{aligned}
K_{0}(x) & =\int_{0}^{\infty} e^{-x \cosh t} d t \leqslant \int_{0}^{\infty} e^{-(x / 2) \exp t} d t=\int_{x / 2}^{\infty} e^{-u} \frac{d u}{u} \\
& \leqslant \int_{x / 2}^{1} \frac{d u}{u}+\int_{1}^{\infty} e^{-u} d u=\log \frac{2}{x}+e^{-1} .
\end{aligned}
$$


Moreover, it is easy to check that $x^{1 / 3}\left(\log (2 / x)+e^{-1}\right)<1.58$ for $x \in(0,1)$. Thus, $K_{0}(x) \leqslant$ $1.58 x^{-1 / 3}$ for $x<1$, and this gives

$$
\left|\widetilde{K}_{i r}(x)\right| \leqslant 2 \cosh \left(\frac{\pi r}{2}\right) K_{0}(x) \leqslant 2 \cosh \left(\frac{\pi}{2}\right) \cdot 1.58 x^{-1 / 3}<8 x^{-1 / 3},
$$

for $r<1$ and $x<1$. We have thus proved that $\left|\widetilde{K}_{i r}(x)\right|<8 x^{-1 / 3}$ for all $r \in \mathbb{R}, x>0$, that is we can take $C=8$ in (A.34).

Acknowledgements. The authors wish to express their thanks to Dennis Hejhal for sharing his unpublished notes [19] and his programs [20] (using steepest descent and numerical integration) for the computation of the $K$-Bessel function.

\section{References}

1. M. Abramowitz and I. A. Stegun (eds), Handbook of mathematical functions with formulas, graphs, and mathematical tables, National Bureau of Standards Applied Mathematics Series 55 (U.S. Government Printing Office, Washington, DC, 1964).

2. R. Aurich, S. Lustig, F. Steiner and H. Then, 'Hyperbolic universes with a horned topology and the cosmic microwave background anisotropy', Classical Quantum Gravity 21 (2004) 4901-4925.

3. C. B. BAlOGH, 'Asymptotic expansions of the modified Bessel function of the third kind of imaginary order', SIAM J. Appl. Math. 15 (1967) 1315-1323.

4. J. Bolte, G. Steil and F. Steiner, 'Arithmetical chaos and violation of universality in energy level statistics', Phys. Rev. Lett. 69 (1992) 2188-2191.

5. A. R. Booker, 'A test for identifying Fourier coefficients of automorphic forms and application to Kloosterman sums', Exper. Math. 9 (2000) 571-581.

6. A. R. Booker and A. Strömbergsson, 'Effective computation of Maass cusp forms', II, in preparation.

7. A. R. Booker, A. Strömbergsson and H. Then, 'Computational details regarding bounds on the K-Bessel function', 2012, Maple file posted on http://www2.math.uu.se/ astrombe/kbessel/compdetails.html.

8. A. R. Booker, A. Strömbergsson and H. Then, 'Software library of some algorithms for rigorous computation of higher transcendental functions', 2012, http://www.maths.bris.ac.uk/ mahlt/software/

9. archt/Boris and E. S. Oran, 'Numerical evaluation of oscillatory integrals such as the modified Bessel function $K_{i \zeta}(x)$ ', J. Comput. Phys. 17 (1975) 425-433.

10. Ll. Closas and J. A. Fernández Rubio, 'Cálculo rápido de las funciones de Bessel modificadas $K_{i s}(X)$ e $I_{i s}(X)$ y sus derivadas', Stochastica 11 (1987) 53-61.

11. A. Cuyt, V. B. Petersen, B. Verdonk, H. Waadeland and W. B. Jones, Handbook of continued fractions for special functions (Springer, 2008).

12. T. M. Dunster, 'Bessel functions of purely imaginary order, with an application to second-order linear differential equations having a large parameter', SIAM J. Math. Anal. 21 (1990) 995-1018.

13. U. T. Ehrenmark, 'The numerical inversion of two classes of Kontorovich-Lebedev transform by direct quadrature', J. Comput. Appl. Math. 61 (1995) 43-72.

14. A. Erdélyi, W. Magnus, F. Oberhettinger and F. G. Tricomi, Higher transcendental functions, vol. II (McGraw-Hill, 1953).

15. A. Gil, J. Segura and N. M. Temme, 'Evaluation of the modified Bessel function of the third kind of imaginary orders', J. Comput. Phys. 175 (2002) 398-411.

16. A. Gil, J. Segura and N. M. Temme, 'Computation of the modified Bessel function of the third kind of imaginary orders: uniform Airy-type asymptotic expansion', J. Comput. Appl. Math. 153 (2003) 225-234.

17. M. Grimmer, 'Interval arithmetic in Maple with intpakX', Proc. Appl. Math. Mech. 2 (2003) 442-443.

18. D. A. Hejhal, The Selberg trace formula for $\operatorname{PSL}(2, R)$, Lecture Notes in Mathematics 1001 (Springer, 1983).

19. D. A. Hejhal, Unpublished notes on the computation of the $K$-Bessel function, 1984 .

20. D. A. Hejhal, 'Eigenvalues of the Laplacian for Hecke triangle groups', Mem. Amer. Math. Soc. 469 (1992) $1-165$.

21. D. A. Hejhal and B. N. Rackner, 'On the topography of Maass waveforms for PSL(2,Z)', Exper. Math. 1 (1992) 275-305.

22. H. IWANIEC, Introduction to the spectral theory of automorphic forms (Rev. Mat. Iberoam., Madrid, 1995).

23. M. K. Kerimov and S. L. Skorokhodov, 'Calculation of modified Bessel functions in the complex domain', Comput. Math. Math. Phys. 24 (1984) 15-24.

24. T. Kiyono and S. Murashima, 'A method of evaluation of the function $K_{i s}(x)$ ', Mem. Fac. Eng. Kyoto Univ. 35 (1973) 102-127.

25. J. D. Lear and J. E. Sturm, 'An integral representation for the modified Bessel function of the third kind, computable for large, imaginary order', Math. Comp. 21 (1967) 496-498. 
26. H. MAAß, 'Über eine neue Art von nichtanalytischen automorphen Funktionen und die Bestimmung Dirichletscher Reihen durch Funktionalgleichungen', Math. Ann. 121 (1949) 141-183.

27. N. Revol and F. Roulllier, 'Motivations for an arbitrary precision interval arithmetic and the MPFI library', Reliable Computing 11 (2005) 275-290, http://perso.ens-lyon.fr/nathalie.revol/software.html.

28. F. W. J. Olver and L. C. Maximon, 'Chapter 10 Bessel functions', NIST Handbook of Mathematical Functions (eds F. W. J. Olver, D. W. Lozier, R. F. Boisvert and C. W. Clark; Cambridge University Press, New York, NY, 2010), http://dlmf.nist.gov/10.

29. F. W. J. Olver, 'Error bounds for asymptotic expansions in turning-point problems', SIAM J. Appl. Math. 12 (1964) 200-214.

30. F. W. J. Olver, Asymptotics and special functions (Academic Press, 1974).

31. The PARI Group, 'PARI/GP, version 2.5.0', Bordeaux, 2011, http://pari.math.u-bordeaux.fr/.

32. W. Shi and R. Wong, 'Hyperasymptotic expansions of the modified Bessel function of the third kind of purely imaginary order', Asymptote. Anal. 63 (2009) 101-123.

33. D. D. Sokolov and A. A. Starobinskit, 'Globally inhomogeneous 'spliced' universes', Sov. Astron. 19 (1976) 629-632.

34. R. L. Stens, 'A unified approach to sampling theorems for derivatives and Hilbert transforms', Signal Process. 5 (1983) 139-151.

35. A. Strömbergsson, 'On the zeros of L-functions associated to Maass waveforms', Int. Math. Res. Not. 1999 (1999) 839-851.

36. G. Szegö, Orthogonal polynomials, American Mathematical Society Colloquium Publications XXIII (American Mathematical Society, Providence, RI, 1939).

37. N. M. Temme, 'Steepest descent paths for integrals defining the modified Bessel functions of imaginary order', Methods Appl. Anal. 1 (1994) 14-24.

38. H. Then, 'Maass cusp forms for large eigenvalues', Math. Comp. 74 (2005) 363-381.

39. H. Then, 'Arithmetic quantum chaos of Maass waveforms', Frontiers in number theory, physics, and geometry I (eds P. Cartier, B. Julia, P. Moussa and P. Vanhove; Springer, 2006), 183-212.

40. H. Then, 'Large sets of consecutive Maass forms and fluctuations in the Weyl remainder', Preprint, 2012, arXiv:1212.3149.

41. I. J. Thompson and A. R. Barnett, 'Coulomb and Bessel functions of complex arguments and order', J. Comput. Phys. 64 (1986) 490-509.

42. G. N. Watson, A treatise on the theory of bessel functions (Cambridge University Press, 1944).

43. P. ZhaO, 'Quantum variance of Maass-Hecke cusp forms', Commun. Math. Phys. 297 (2010) 475-514.

\author{
Andrew R. Booker \\ Department of Mathematics \\ University of Bristol \\ University Walk \\ Bristol BS8 1TW \\ United Kingdom \\ andrew.booker@bristol.ac.uk \\ Holger Then \\ Department of Mathematics \\ University of Bristol \\ University Walk \\ Bristol BS8 1TW \\ United Kingdom \\ holger.then@bristol.ac.uk
}

\author{
Andreas Strömbergsson \\ Department of Mathematics \\ Box 480, Uppsala University \\ S-75106 Uppsala \\ Sweden
}

andreas.strombergsson@math.uu.se 